TRANSACTIONS OF THE

AMERICAN MATHEMATICAL SOCIETY

Volume 364, Number 1, January 2012, Pages 137-174

S 0002-9947(2011)05354-7

Article electronically published on August 25, 2011

\title{
SIMPLE VECTOR BUNDLES ON PLANE DEGENERATIONS OF AN ELLIPTIC CURVE
}

\author{
LESYA BODNARCHUK, YURIY DROZD, AND GERT-MARTIN GREUEL
}

\begin{abstract}
In 1957 Atiyah classified simple and indecomposable vector bundles on an elliptic curve. In this article we generalize his classification by describing the simple vector bundles on all reduced plane cubic curves. Our main result states that a simple vector bundle on such a curve is completely determined by its rank, multidegree and determinant. Our approach, based on the representation theory of boxes, also yields an explicit description of the corresponding universal families of simple vector bundles.
\end{abstract}

\section{INTRODUCTION}

The theory of vector bundles on an elliptic curve and its degenerations is known to be closely related with the theory of integrable systems (see e.g. Kri77, Ma78, Mu94). Another motivation for studying vector bundles on elliptic fibrations comes from the work of Friedman, Morgan and Witten [FMW99, who discovered their importance for heterotic string theory. The main motivation of our investigation was the following problem. Let $\mathbb{E} \rightarrow T$ be an elliptic fibration, where $T$ is some basis such that for any point $t \in T$ the fiber $\mathbb{E}_{t}$ is a reduced projective curve with the trivial dualizing sheaf.

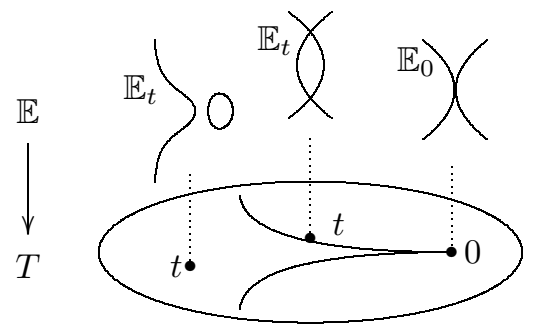

In most applications, a generic fiber of this fibration is an elliptic curve and for the points of the discriminant locus $\Delta \subset T$ the fibers are singular (and possibly reducible). Can one give a uniform description of simple vector bundles both on the smooth and the singular fibers?

Received by the editors July 28, 2009 and, in revised form, March 3, 2010.

2000 Mathematics Subject Classification. Primary 16G60; Secondary 14H10 and 14H60.

Key words and phrases. Simple vector bundles and their moduli, degeneration of an elliptic curve, tame and wild, small reduction.

We express our sincere thanks to Professor Serge Ovsienko for fruitful discussions and helpful advice. The first named author would like to thank Institut des Hautes Études Scientifiques and the Mathematisches Forschungsinstitut Oberwolfach, where she stayed during the period that this paper was written.

(C)2011 American Mathematical Society Reverts to public domain 28 years from publication 
It is known that the category of all vector bundles of a singular genus one curve $E$ essentially depends on the singularity type of the curve. For example, in the case of the Weierstraß family $\mathbb{E} \rightarrow \mathbb{C}^{2}$ given by the equation $z y^{2}=4 x^{3}+g_{2} x z^{2}+g_{3} z^{3}$, the cuspidal fiber $E=\mathbb{E}_{(0,0)}$ is vector-bundle-wild, whereas all the other fibers $\mathrm{E}=\mathbb{E}_{\left(g_{2}, g_{3}\right)}$ (smooth and nodal) are vector-bundle-tame 1 This phenomenon seems to be rather strange, since very strong continuity results for the Picard functor are known to be true AK79. It is one of the results of this paper that the situation is completely different if one restricts to the study of the simple 2 vector bundles. Namely we prove that the category $\mathrm{VB}_{E}^{s}$ of simple vector bundles on $E$ is indeed tame. Moreover, we provide a complete classification of simple bundles and describe a bundle on the moduli space, having certain universal properties.

The starting point of our investigation and the main source of inspiration was the following classical result of Atiyah.

Theorem 1.1 ([Ati57]). Let $E$ be an elliptic curve over an algebraically closed field $\mathbb{k}$. Then a simple vector bundle $\mathcal{E}$ on $E$ is uniquely determined by its rank $r$ and degree $d$, which should be coprime, and determinant $\operatorname{det}(\mathcal{E}) \in \operatorname{Pic}^{d}(E) \cong E$.

The main result of our article generalizes Atiyah's theorem to all reduced plane degenerations of an elliptic curve. Singular fibers of elliptic fibred surfaces were described by Kodaira and throughout this article we make use of his classification; see for example BPV84, Table 3, p.150]. In what follows the cycles of projective lines (also called Kodaira cycles) are denoted by $\mathrm{I}_{N}$, where $N$ is the number of irreducible components. Note that a Kodaira cycle $\mathrm{I}_{N}$ is a plane curve if and only if $N \leq 3$. Besides them, there are precisely three other Kodaira fibers. Thus, we study simple vector bundles on the six configurations in Table 1 .

TABLE 1

\begin{tabular}{|c|c|c|}
\hline$N$ & Kodaira cycles & Kodaira fibers \\
\hline$N=1$ & $\mathrm{I}_{1}: \quad y^{2} z=x^{3}+x^{2} z$ & II : $\quad y^{2} z=x^{3}$ \\
\hline$N=2$ & $\mathrm{I}_{2}: \quad z^{3}=$ & III : $\quad y^{2} z=x^{2} y$ \\
\hline$N=3$ & $\mathrm{I}_{3}: x y z$ & IV $: \quad x y^{2}=x^{2} y$ \\
\hline
\end{tabular}

\footnotetext{
${ }^{1}$ In representation theory a category is called tame if its indecomposable objects can be described by some discrete and one continuous parameters, and wild if they are non-classifiable. An algebraic variety $X$ is called vector-bundle-wild or vector-bundle-tame if the category $\mathrm{VB}_{X}$ of vector bundles on $X$ is wild or respectively tame (see DG01).

${ }^{2} \mathrm{~A}$ bundle is called simple if it admits no endomorphisms but homotheties.
} 
In order to present our main theorem, let us fix some notation. Throughout this article, let $\mathbb{k}$ be an algebraically closed field and a curve be a reduced projective curve. Let $E$ be a plane degeneration of an elliptic curve, $N=1,2,3$ the number of its irreducible components and $L_{k}$ the $k$-th component of $E$. For a vector bundle $\mathcal{E}$ on $E$ we denote by

- $d_{k}=d_{k}(\mathcal{E})=\operatorname{deg}\left(\left.\mathcal{E}\right|_{L_{k}}\right) \in \mathbb{Z}$ the degree of the restriction of $\mathcal{E}$ on $L_{k}$;

- $\mathbb{d}=\mathbb{d}(\mathcal{E})=\left(d_{1}, \ldots, d_{N}\right) \in \mathbb{Z}^{N}$ the multidegree of $\mathcal{E}$

- $d=\operatorname{deg}(\mathcal{E})=d_{1}+\cdots+d_{N}$ the degree of $\mathcal{E}$. In our cases it is equal to the Euler-Poincaré characteristic: $\chi(\mathcal{E})=h^{0}(\mathcal{E})-h^{1}(\mathcal{E})$.

- $r=\operatorname{rank}(\mathcal{E})=$ the rank of $\mathcal{E}$.

Moreover, let $\mathrm{Pic}^{\mathrm{d}}(E)^{3}$ be the Picard group of invertible sheaves of multidegree $\mathbb{d}$ on $E$. The following theorem generalizes Atiyah's classification and is the main result of this article.

Theorem 1.2. Let $E$ be a reduced plane cubic curve with $N$ irreducible components, $1 \leq N \leq 3$.

(i) Then the rank $r$ and the degree $d$ of a simple vector bundle on $E$ are coprime. For any tuple of integers $(r, \mathbb{d}) \in \mathbb{N} \times \mathbb{Z}^{N}$ such that $\operatorname{gcd}\left(r, d_{1}+\cdots+\right.$ $\left.d_{N}\right)=1$, let $\mathscr{M}=\mathrm{VB}_{E}^{s}(r, \mathbb{d})$ be the set of simple vector bundles of rank $r$ and multidegree $\mathbb{d}$. Then the map $\operatorname{det}: \mathscr{M} \rightarrow \operatorname{Pic}^{\mathbb{d}}(E)$ is a bijection.

(ii) The Jacobian $\mathrm{Pic}^{(0, \ldots, 0)}(E)$ acts transitively on $\mathscr{M}$. The stabilizer of a point is isomorphic to $\mathbb{Z}_{r}$ if $E$ is a Kodaira cycle and is trivial in the remaining cases.

Let $\Lambda:=\mathbb{k}^{*}$ if $E$ is a Kodaira cycle and $\Lambda:=\mathbb{k}$ if $E$ is a Kodaira fiber of type II, III or IV. By 1.2(i), $\Lambda$ is a moduli space of simple vector bundles of given rank $r$ and multidegree $\mathbb{d}$ provided $\operatorname{gcd}(r, d)=1$. By an observation of Burban and Kreußler [BK4, for a given tuple of integers $(r, \mathbb{d}) \in \mathbb{N} \times \mathbb{Z}^{N}$ such that $\operatorname{gcd}(r, d)=1$, our method yields an explicit construction of a vector bundle $\mathcal{P}=\mathcal{P}(r, \mathbb{d}) \in \mathrm{VB}_{E \times \Lambda}$ satisfying in the general case only the following universality properties:

- for any point $\lambda \in \Lambda$ the vector bundle $\mathcal{P}(\lambda):=\left.\mathcal{P}\right|_{E \times\{\lambda\}} \in \operatorname{VB}(E)$ is simple of rank $r$ and multidegree $\mathbb{d}$;

- for any vector bundle $\mathcal{E} \in \mathrm{VB}_{E}^{s}(r, \mathbb{d})$ there exists a unique $\lambda \in \Lambda$ such that $\mathcal{E} \cong \mathcal{P}(\lambda)$

- for two points $\lambda \neq \mu$ from $\Lambda$ we have $\mathcal{P}(\lambda) \neq \mathcal{P}(\mu)$.

If the curve $E$ is irreducible, the vector bundle $\mathcal{P}$ is the universal family of stable vector bundles of rank $r$ and degree $d$.

Similarly to Atiyah's proof Ati57, the main ingredient of our approach is a construction of various bijections $\operatorname{VB}_{E}^{s}(r, \mathbb{d}) \rightarrow \operatorname{VB}_{E}^{s}\left(r^{\prime}, \mathbb{d}^{\prime}\right)$, where $r^{\prime}<r$. However, our method is completely different from Atiyah's. We use a reduction of our classification problem to the description of bricks in the category of representations of a certain box (or a differential biquiver). Moreover, we provide an explicit algorithm (Algorithm (7.2) that for a given tuple $(r, \mathbb{d}) \in \mathbb{N} \times \mathbb{Z}^{N}$ constructs a canonical form

\footnotetext{
${ }^{3}$ Note that $\mathrm{Pic}^{\mathbb{d}}(E)$ is $E$ for an elliptic curve, $\mathbb{k}^{*}$ for Kodaira cycles and $\mathbb{k}$ for the other Kodaira fibers.
} 
of a matrix, describing the universal family of simple vector bundles of rank $r$ and multidegree $\mathbb{d}$. The core of this algorithm is the automaton of reduction, which is given for each of the listed curves and operates on discrete parameters like the Euclidean algorithm.

For a rather long time (till the middle of the 1970s) there were no efficient methods for studying moduli spaces of vector bundles of higher ranks on singular curves. In order to study vector bundles on (possibly reducible) projective curves with only nodes or cusps as singularities, Seshadri introduced the concept of the socalled parabolic bundles (see [Ses82, Section 3]). This approach was later developed by Bhosle, who introduced the notion of generalized parabolic bundles Bho92, Bho96.

Our method of studying vector bundles on genus one curves is a certain categorification of the language of parabolic bundles of Seshadri and Bhosle. It was originally proposed in DG01]; see also BDG01] and BBDG] for some further elaborations. The idea of this method can be explained as follows. Let $X$ be a singular reduced projective curve (typically rational, but with arbitrary singularities), $\pi: \widetilde{X} \rightarrow X$ its normalization. Then a description of the fibers of the functor $\pi^{*}: \mathrm{VB}_{X} \rightarrow \mathrm{VB}_{\tilde{X}}$ can be converted to some representation theory problem, called a matrix problem. The main application of this method concerns the case of curves of arithmetic genus one. In the case of a cycle of $N$ projective lines (Kodaira cycles $I_{N}$ ), the obtained matrix problem turns out to be representation-tame; see Bon92 and CB89. As a result, it allows us to obtain a complete classification of indecomposable torsion-free sheaves on these genus one curves; see [DG01] and BBDG].

However, a description of the exact combinatorics of simple vector bundles on a cycle of projective lines requires some extra work. This was done in BDG01, but the resulting answer was not very explicit. For the case of a nodal cubic curve $z y^{2}=x^{3}+x^{2} z$, in Bur03, Burban derived the statement of Theorem 1.2 using the classification of all indecomposable objects. In this article we give an improved description of simple vector bundles on cycles $\mathrm{I}_{1}, \mathrm{I}_{2}$ and $\mathrm{I}_{3}$ using the technique of the so-called small reductions of matrix problems.

As we have mentioned above, the representation-theoretic properties of the category of torsion-free sheaves on Kodaira cycles and the other degenerations of elliptic curves are rather different. For example, for a cuspidal rational curve $z y^{2}=x^{3}$, even the classification of indecomposable semi-stable vector bundles of a given slope is a representation-wild problem. However, if we additionally impose the simplicity assumption, then the wild fragments of the matrix problem disappear and we can reduce the matrices to a canonical form (see BD03]).

The matrix problems describing simple vector bundles on nodal and cuspidal cubic curves are relatively easy to deal with, since they are self-reproducing; i.e., after applying one step of small reduction we obtain the same problem but with matrices of smaller sizes. In fact, the matrix reduction operates on discrete parameters of vector bundles as the Euclidean algorithm. Carrying this out we obtain the statement of Theorem 1.2 for irreducible degenerations of an elliptic curve. Unfortunately, the matrix problems for curves with many components are no longer self-reproducing. However, they turn out to be such in some bigger class of matrix problems. To study this class in a conceptual way we need more sophisticated methods from representation theory. Namely, we describe our matrix problem as 
the category of representations of a certain box (also called bocs, "bimodule over a category with a coalgebra structure" or differential biquiver); see [Bod07, BD09].

The technique of boxes is known to be very useful for proving tame-wild dichotomy theorems and various semi-continuity results; see [Dro79, Dro01, Dro05, CB90, etc. A new feature, illustrated in this article, is that the formalism of boxes can be very efficiently applied for constructing canonical forms of representations "in general position". A usual approach to a matrix problem is a consecutive application of a minimal edge reduction, which is a reduction of a certain block to its Gauß form. However, since we are interested in bricks it turns out that it is sufficient to take into account only small reductions, which are Gauß reductions provided that the rank of the block is maximal. This way for each plane singular cubic curve and the matrix problem corresponding to the family of simple vector bundles of rank $r$ and multidegree $\mathbb{d}$ we get an explicit algorithm constructing its canonical form. The course of the construction is given as a path on some automaton, whose states are boxes and transition arrows are small reductions.

To put our results in a broader mathematical context we would like to mention that the case of singular curves of genus one is special in many respects. We are especially interested in the study of vector bundles on curves having trivial dualizing bundle. This automatically implies that they have arithmetic genus one, but not vice versa. In FMW99, Friedman, Morgan and Witten proposed a powerful method of constructing vector bundles on irreducible genus one curves and elliptic fibrations, based on the technique of the so-called spectral covers. Later, it was realized that their construction can be alternatively described using the language of Fourier-Mukai transforms; see e.g. [BK05, BBHM02, HLSP. Although for irreducible cubic curves Theorem 1.2 was previously known and can be proven using either geometric invariant theory or Fourier-Mukai transforms, our approach has one particular advantage. Namely, it yields a very explicit description of a universal family of simple vector bundles, which turned out to be important in applications. In particular, it was used to obtain new solutions of the associative and quantum Yang-Baxter equations; see [Pol07] and [BK4, Section 9].

We should also mention that the geometric point of view suggests replacing the simplicity condition by Simpson stability. Despite the fact that for reducible curves there are even line bundles which are not Simpson semi-stable, both notions are closely related for curves of arithmetic genus one. In Lo05] and Lo06, LópezMartin described the geometry of the compactified Jacobian in the case of Kodaira fibers and elliptic fibrations.

Organization of the material. In Section 2 we recall the construction of [DG01] and replace the category of vector bundles $\mathrm{VB}_{E}$ by the equivalent category of triples $\operatorname{Tr}_{E}$. Fixing bases of triples we obtain the category of matrices $M P_{E}$. In Sections 3 and 4 this procedure is applied to all the curves from Table1, In Section 5 we study the properties induced by the simplicity condition and obtain some additional restrictions for the matrix problem $\mathrm{MP}_{E}$. In Section 6 we fix discrete parameters $(r, \mathbb{d})$ and reduce a brick object 4 of $\mathrm{MP}_{E}(r, \mathbb{d})$ to its partial canonical form. Remarkably, this new matrix problem and its dimension vector $\$$ are completely determined by

\footnotetext{
${ }^{4} \mathrm{~A}$ brick or a schurian object is a representation with no nonscalar endomorphisms.
} 
the curve $E$, the rank $r$ and the multidegree $\mathbb{d}$. This correspondence for the curves with many components is given in Tables $2-4$.

In Section 7 we provide a formal approach to the partially reduced matrix problem: we interpret it as the category of bricks $\operatorname{Br}_{\mathfrak{A}}(\mathbb{s})$ of some box $\mathfrak{A}$ and dimension vector $\$$. We prove that any break is a module in a general position; thus the Gauß reduction can be replaced by the small one. A course of reduction can be presented as a path on some automaton, where the states are matrix problems and transitions are small reductions. We call a box principal if $\operatorname{Br}_{\mathfrak{A}}(\mathbb{\$}) \cong \operatorname{VB}_{E}^{s}(r, \mathbb{d})$. For a fixed rank $r$ and multidegree $\mathbb{d}$, if the set $\mathrm{Br}_{\mathfrak{A}}(\mathbb{S})$ is nonempty, then there is a path $p: \mathfrak{A} \rightarrow \mathfrak{A}^{\prime}$, where $\mathfrak{A}^{\prime}$ is principal, reducing the dimension vector $\$$ to $(1,0, \ldots, 0)$ :

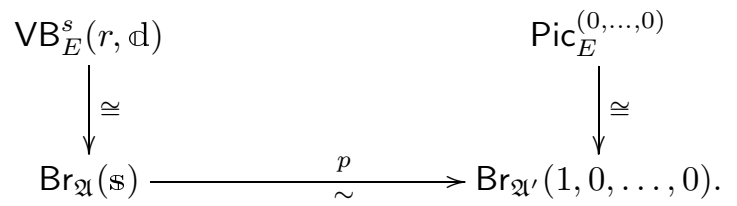

A transition operates on the pair $(d \bmod r, r-d \bmod r)$ as the Euclidean algorithm and for $\mathcal{E} \in \mathrm{VB}_{E}^{s}(r, \mathbb{d})$ we obtain $\operatorname{gcd}(r, d)=1$. It turns out that this condition is not only necessary but also sufficient for $\mathrm{VB}_{E}^{s}(r, \mathbb{d})$ to be nonempty. The canonical form of a brick from $\mathrm{Br}_{\mathfrak{A}}(\mathrm{s})$ can be recovered by reversing the path $p$. The whole procedure is emphasized in Algorithm 7.2 .

In Sections 8 - 10 we construct automatons for each Kodaira cycle $I_{N}(N \leq 3)$ and show that a path on it also encodes a course of reduction for the Kodaira fiber with $N$ components.

Analyzing how a path operates on the dimension vector $\$$ we deduce the first part of Theorem 1.2. In Section [1] we illustrate Algorithm [7.2 on some concrete examples. In Section 12 we describe the action of $\operatorname{Pic}^{(0, \ldots, 0)}(E)$ on $\operatorname{VB}_{E}^{s}(r, \mathbb{d})$ and morphisms between simple bundles, thus deducing the second part of Theorem 1.2 .

\section{General approach}

Category of triples. Let $\mathbb{k}$ be an algebraically closed field 5 Sch $:=$ Sch $/ \mathbb{k}$ the category of Noetherian schemes over $\mathbb{k}$ and for any scheme $T \in \mathrm{Sch}$ by $\mathrm{VB}_{T}, \mathrm{TF}_{T}$ and $\mathrm{Coh}_{T}$ we denote the categories of vector bundles, torsion-free coherent and coherent sheaves on $T$ respectively.

Let $X$ be a singular curve over $\mathbb{k}$. Fix the following notation:

- $\pi: \widetilde{X} \longrightarrow X$ the normalization of $X$

- $\mathcal{O}:=\mathcal{O}_{X}$ and $\widetilde{\mathcal{O}}:=\mathcal{O}_{\widetilde{X}}$ the structure sheaves of $X$ and $\widetilde{X}$ respectively;

- $\mathcal{J}=\operatorname{Ann} n_{\mathcal{O}}\left(\pi_{*} \widetilde{\mathcal{O}} / \mathcal{O}\right)$ the conductor of $\mathcal{O}$ in $\pi_{*} \widetilde{\mathcal{O}}$

- $\imath: S \hookrightarrow X$ the subscheme of $X$ defined by the conductor $\mathcal{J}$ and $\tilde{\imath}: \widetilde{S} \hookrightarrow \widetilde{X}$ its scheme-theoretic pull-back to the normalization $\widetilde{X}$.

\footnotetext{
${ }^{5}$ Although the construction of triples and many classification results are valid for an arbitrary field, the matrix problems that we obtain can be quite special and require different methods to deal with. In order to get a uniform description for all cases we assume from the beginning the ground field $\mathbb{k}$ to be algebraically closed.
} 
Altogether they fit into a Cartesian diagram:

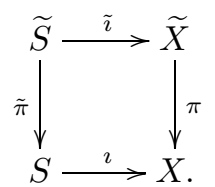

Remark 2.1. 1. In what follows we shall identify the structure sheaf $\mathcal{O}_{T}$ of an Artinian scheme $T$ with the coordinate $\operatorname{ring} \mathbb{k}[T]$.

2. The main property of the conductor is that for the ideal $\widetilde{\mathcal{J}}:=\mathcal{I}_{\widetilde{S}}$ in $\widetilde{\mathcal{O}}$ we have $\mathcal{J}=\pi_{*} \widetilde{\mathcal{J}}$.

3. Let $\mathcal{F} \in \operatorname{Coh}_{X}$ and $\widetilde{\mathcal{F}} \in \operatorname{Coh}_{\widetilde{X}}$ be coherent sheaves on $X$ and $\widetilde{X}$ respectively. With a little abuse of notation one can write: $\imath^{*} \mathcal{F}=\mathcal{F} \otimes_{\mathcal{O}} \mathcal{O}_{S}=\mathcal{F} / \mathcal{J F} \in$ $\operatorname{Coh}_{S}$ and $\tilde{\imath}^{*} \widetilde{\mathcal{F}}=\widetilde{\mathcal{F}} \otimes_{\widetilde{\mathcal{O}}} \mathcal{O}_{\widetilde{S}}=\widetilde{\mathcal{F}} / \widetilde{\mathcal{J}} \widetilde{\mathcal{F}} \in \operatorname{Coh}_{\widetilde{S}}$. Since $S$ and $\widetilde{S}$ are schemes of dimension zero, $\imath_{*} \imath^{*} \mathcal{F}$ and $\tilde{\imath}_{*} \tau^{*} \widetilde{\mathcal{F}}$ are skyscraper sheaves on $X$ and $\widetilde{X}$ respectively.

The usual way to deal with vector bundles on a singular curve is to lift them to the normalization and work on a smooth curve; see for example [Ses82, Bho92, Bho96]. To describe the fibers of the map $\mathrm{VB}_{X} \rightarrow \mathrm{VB}_{\tilde{X}}$ we recall the following construction:

Definition 2.2. The category of triples $\operatorname{Tr}_{X}$ is defined as follows:

- Its objects are triples $(\widetilde{\mathcal{F}}, \mathcal{M}, \tilde{\mu})$, where $\widetilde{\mathcal{F}} \in \mathrm{VB}_{\tilde{X}}, \mathcal{M} \in \mathrm{VB}_{S}$ and $\tilde{\mu}$ : $\tilde{\pi}^{*} \mathcal{M} \rightarrow \tilde{\imath}^{*} \widetilde{\mathcal{F}}$ is an isomorphism of $\mathcal{O}_{\widetilde{S}}$-modules.

- A morphism $(\widetilde{\mathcal{F}}, \mathcal{M}, \tilde{\mu}) \stackrel{(F, f)}{\longrightarrow}\left(\widetilde{\mathcal{F}}^{\prime}, \mathcal{M}^{\prime}, \tilde{\mu}^{\prime}\right)$ is given by a pair $(F, f)$, where $F: \widetilde{\mathcal{F}} \rightarrow \widetilde{\mathcal{F}}^{\prime}$ is a morphism in $\operatorname{VB}_{\widetilde{X}}$ and $f: \mathcal{M} \rightarrow \mathcal{M}^{\prime}$ is a morphism in $\mathrm{Coh}_{S}$, such that the following diagram commutes in $\operatorname{Coh}_{\widetilde{S}}$ :

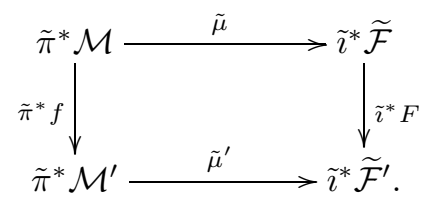

The raison d'être for the formalism of triples is the following theorem:

Theorem 2.3 (DG01). The functor $\Psi: \mathrm{VB}_{X} \longrightarrow \operatorname{Tr}_{X}$ taking a vector bundle $\mathcal{F}$ to the triple $(\widetilde{\mathcal{F}}, \mathcal{M}, \tilde{\mu})$, where $\widetilde{\mathcal{F}}:=\pi^{*} \mathcal{F}, \mathcal{M}:=\imath^{*} \mathcal{F}$ and $\tilde{\mu}$ is the canonical morphism $\tilde{\mu}: \tilde{\pi}^{*} \imath^{*} \mathcal{F} \rightarrow \tilde{\imath}^{*} \pi^{*} \mathcal{F}$, is an equivalence of categories.

Although the statement of Theorem 2.3 holds for arbitrary reduced curves, the method based on it can be efficiently used mainly for rational curves, since in this case the description of vector bundles on the normalization is well understood.

Vector bundles on a projective line. According to the classical result known as the Birkhoff-Grothendieck Theorem, a vector bundle $\widetilde{\mathcal{F}}$ on a projective line $\mathbb{P}^{1}$ 
splits into a direct sum of line bundles:

$$
\widetilde{\mathcal{F}} \cong \bigoplus_{n \in \mathbb{Z}}\left(\mathcal{O}_{\mathbb{P}^{1}}(n)\right)^{r_{n}}
$$

Let $\left(z_{0}: z_{1}\right)$ be homogeneous coordinates on $\mathbb{P}^{1}$. Then an endomorphism $F$ of $\widetilde{\mathcal{F}}$ can be written in a matrix form:

$$
F=\left(\begin{array}{ccccc}
\ddots & 0 & \ldots & 0 & 0 \\
\cdots & F^{n n} & \ldots & 0 & 0 \\
& \vdots & \ddots & \vdots & \vdots \\
\ldots & F^{m n} & \ldots & F^{m m} & 0 \\
& \vdots & & \vdots & \ddots
\end{array}\right),
$$

where $F^{m n}$ are blocks of sizes $r_{m} \times r_{n}$ with coefficients in the vector space

$$
\operatorname{Hom}_{\mathbb{P}^{1}}\left(\mathcal{O}_{\mathbb{P}^{1}}(n), \mathcal{O}_{\mathbb{P}^{1}}(m)\right) \cong \mathbb{k}\left[z_{0}, z_{1}\right]_{m-n},
$$

since a morphism $\mathcal{O}_{\mathbb{P}^{1}}(n) \rightarrow \mathcal{O}_{\mathbb{P}^{1}}(m)$ is determined by a homogeneous form $Q\left(z_{0}, z_{1}\right)$ of degree $m-n$. In particular, the matrix $F$ is lower-block-triangular and the diagonal $r_{n} \times r_{n}$ blocks $F^{n n}$ are matrices over $\mathbb{k}$. The morphism $F$ is an isomorphism if and only if all the diagonal blocks $F^{n n}$ are invertible.

Matrix problem $M P_{X}$. To classify vector bundles on a rational projective curve $X$ with the normalization $\widetilde{X}=\bigsqcup_{k=1}^{N} L_{k}$ one should describe iso-classes of objects in $\operatorname{Tr}_{X}$. Note that two triples $(\widetilde{\mathcal{F}}, \mathcal{M}, \tilde{\mu})$ and $\left(\widetilde{\mathcal{F}}^{\prime}, \mathcal{M}^{\prime}, \tilde{\mu}^{\prime}\right)$ are isomorphic only if $\widetilde{\mathcal{F}} \cong \widetilde{\mathcal{F}}^{\prime}$ and $\mathcal{M} \cong \mathcal{M}^{\prime}$. By the Birkhoff-Grothendieck Theorem a bundle $\widetilde{\mathcal{F}}$ on $\widetilde{X}$ can be given by a tuple of integers $\mathbb{r}:=\{r(n, k)\}$, where $n \in \mathbb{Z}, 1 \leq k \leq N$ and $\sum_{n \in \mathbb{Z}} r(n, k)=r$ for each $k$. Let $\mathrm{MP}_{X}:=\bigcup_{\mathbb{r}} \mathrm{MP}_{X}(\mathbb{r})$ be the following KrullSchmidt category: an object of a stratum $\operatorname{MP}_{X}(\mathbb{r})$ is a matrix $\tilde{\mu}$ for which there exists a triple $(\widetilde{\mathcal{F}}, \mathcal{M}, \tilde{\mu}) \in \operatorname{Tr}_{X}$ and the vector bundle $\widetilde{\mathcal{F}} \in \mathrm{VB}_{\widetilde{X}}$ splits into a direct sum of line bundles with the tuple of multiplicities $\mathbb{r}$. For two objects $\tilde{\mu}$ and $\tilde{\mu}^{\prime}$ with triples $(\widetilde{\mathcal{F}}, \mathcal{M}, \tilde{\mu})$ and $\left(\tilde{\mathcal{F}}^{\prime}, \mathcal{M}^{\prime}, \tilde{\mu}^{\prime}\right)$, respectively, a morphism from $\tilde{\mu}$ to $\tilde{\mu}^{\prime}$ is a pair $\left(\tilde{\imath}^{*} F, \tilde{\pi}^{*} f\right)$ such that $\tilde{\imath}^{*} F \cdot \tilde{\mu}=\tilde{\mu}^{\prime} \cdot \tilde{\pi}^{*} f$, where $F \in \operatorname{Hom}_{\tilde{X}}\left(\widetilde{\mathcal{F}}, \widetilde{\mathcal{F}}^{\prime}\right)$ and $f \in \operatorname{Hom}\left(\mathcal{M}, \mathcal{M}^{\prime}\right)$. The functor $\mathrm{H}: \operatorname{Tr}_{X} \longrightarrow \mathrm{MP}_{X}$ is full and dense and there is a natural projection

$$
\operatorname{Hom}_{\operatorname{Tr}_{X}}\left((\widetilde{\mathcal{F}}, \mathcal{M}, \tilde{\mu}),\left(\widetilde{\mathcal{F}}, \mathcal{M}, \tilde{\mu}^{\prime}\right)\right) \rightarrow \operatorname{Hom}_{\mathrm{MP}_{X}}\left(\tilde{\mu}, \tilde{\mu}^{\prime}\right) .
$$

Definition 2.4. Replacing the set of morphisms by the set of invertible morphisms in $\mathrm{MP}_{X}(\mathrm{r})$ (also called matrix transformations) we obtain some groupoid. A matrix problem is the problem of describing orbits of indecomposable objects. If it is possible, a solution consists in finding a canonical form of $\tilde{\mu}$.

The precise description of this procedure can be found in Bod07. For convenience we choose $\mathbb{k}$-bases of $\mathcal{O}_{S}$ and $\mathcal{O}_{\widetilde{S}}$ and rewrite $\tilde{\mu}, \tilde{\imath}^{*} F$ and $\tilde{\pi}^{*} f$ as tuples of matrices over $\mathbb{k}$. 


\section{MATRIX PROBLEM FOR CYCLES OF PROJECTIVE LINES}

Let $E$ be a cycle of $N$ projective lines. The normalization $\widetilde{E}$ is a disjoint union of $N$ copies of $\mathbb{P}^{1}$. For example, for $N=3$ we have:

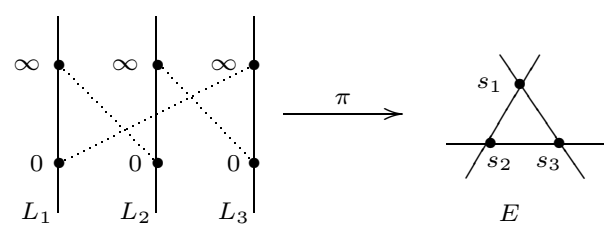

Let $s_{1}, \ldots, s_{N}$ be the intersection points ordered in such a way that $s_{k}$ and $s_{k+1}$ belong to the component $L_{k}$ for $k=1, \ldots, N-1$ and the points $s_{N}$ and $s_{1}$ lie on $L_{N}$. On each component $L:=L_{k}$ choose the local coordinates such that the preimages of $s_{k}$ and $s_{k+1}$ on $L_{k}$, for $k=1, \ldots, N-1$, and $s_{N}$ and $s_{1}$ on $L_{N}$ have coordinates $0:=(0: 1)$ and $\infty:=(1: 0)$. Then

$$
\mathcal{O}_{S}=\mathbb{k}\left(s_{1}\right) \oplus \cdots \oplus \mathbb{k}\left(s_{N}\right) \text { and } \mathcal{O}_{\widetilde{S}}=\bigoplus_{k=1}^{N}\left(\mathbb{k}\left(0_{k}\right) \oplus \mathbb{k}\left(\infty_{k}\right)\right) .
$$

To obtain the matrix problem $\mathrm{MP}_{E}$ we fix:

- a splitting $\widetilde{\mathcal{F}} \cong \bigoplus_{k=1}^{N}\left(\bigoplus_{n \in \mathbb{Z}} \mathcal{O}_{L_{k}}(n)^{r(n, k)}\right)$ with $\sum_{n \in \mathbb{Z}} r(n, k)=r$ for each component $k$;

- an isomorphism $\mathcal{M} \cong \mathcal{O}_{S}^{r}=\left(\bigoplus_{k=1}^{N} \mathbb{k}\left(s_{k}\right)\right)^{r}$.

- The choice of coordinates on each component $L$ of $\tilde{X}$ fixes two canonical sections $z_{0}$ and $z_{1}$ of $H^{0}\left(\mathcal{O}_{L}(1)\right)$, and we use the following trivializations:

$$
\begin{aligned}
& \mathcal{O}_{L}(n) \otimes \mathcal{O}_{L \cap \widetilde{S}} \stackrel{\sim}{\longrightarrow} \mathbb{k}(0) \times \mathbb{k}(\infty) \\
& \zeta \otimes 1 \longmapsto\left(\zeta / z_{1}^{n}(0), \zeta / z_{0}^{n}(\infty)\right) .
\end{aligned}
$$

This isomorphism only depends on the choice of coordinates on $L \cong \mathbb{P}^{1}$. In such a

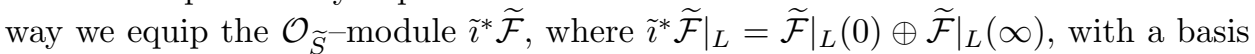
and get isomorphisms $\left.\widetilde{\mathcal{F}}\right|_{L}(0) \cong \mathbb{k}(0)^{r}$ and $\left.\widetilde{\mathcal{F}}\right|_{L}(\infty) \cong \mathbb{k}(\infty)^{r}$.

Matrix problem $\mathrm{MP}_{E}$ for Kodaira cycles $I_{N}$. With respect to all the choices, the maps $\tilde{\mu}, \tilde{\imath}^{*} F$ and $\tilde{\pi}^{*} f$ can be written as matrices.

- The gluing map $\tilde{\mu}: \tilde{\pi}^{*} \mathcal{M} \stackrel{\sim}{\longrightarrow} \tilde{\imath}^{*} \widetilde{\mathcal{F}}$ consists of $2 N$ invertible matrices over $\mathrm{k}$ :

$$
\tilde{\mu}=\left(\mu_{1}(0), \mu_{1}(\infty), \mu_{2}(0), \mu_{2}(\infty), \ldots, \mu_{N}(0), \mu_{N}(\infty)\right) .
$$

- If we have a morphism $\mathcal{O}_{L}(n) \rightarrow \mathcal{O}_{L}(m)$ given by a homogeneous form $Q\left(z_{0}, z_{1}\right)$ of degree $m-n$, then it induces a map $\mathcal{O}_{L}(n) \otimes \mathcal{O}_{\widetilde{S}} \longrightarrow \mathcal{O}_{L}(m) \otimes \mathcal{O}_{\widetilde{S}}$ given by $(Q(0), Q(\infty)):=(Q(0: 1), Q(1: 0))$. Hence, with respect to the chosen trivializations of $\mathcal{O}_{L}(n)$ at 0 and $\infty$ the map

$$
\left.\tilde{\imath}^{*} F\right|_{L}=\left(F_{k}(0), F_{k}(\infty)\right): \mathbb{k}^{r}(0) \oplus \mathbb{k}^{r}(\infty) \longrightarrow \mathbb{k}^{r}(0) \oplus \mathbb{k}^{r}(\infty)
$$

is given by a pair of lower block triangular matrices $\left(F_{k}(0), F_{k}(\infty)\right)$ consisting of blocks $F_{k}^{m n}(0), F_{k}^{m n}(\infty) \in \operatorname{Mat}_{\mathbb{k}}(r(m, k) \times r(n, k))$, for $m>n$ and 
with common diagonal blocks $F_{k}^{n n} \in \operatorname{Mat}_{\mathbb{k}}(r(n, k) \times r(n, k))$. The morphism $F$ is invertible if all the diagonal blocks $F_{k}^{n n}$ belong to GL( $(k, r(n, k))$.

- The same holds for the induced map $\tilde{\pi}^{*} f=\left(f_{1}, \ldots, f_{N}\right)$ : if $(F, f)$ is invertible, then $f_{k} \in \mathrm{GL}(\mathbb{k}, r)$ for each component $k$.

- The transformation rule $\tilde{\mu} \mapsto\left(\tilde{\imath}^{*} F\right) \cdot \tilde{\mu} \cdot\left(\tilde{\pi}^{*} f\right)^{-1}$ can be rewritten for each component $k$ as $\mu_{k}(0) \mapsto F_{k}(0) \mu_{k}(0) f_{k}^{-1}$ and $\mu_{k}(\infty) \mapsto F_{k}(\infty) \mu_{k+1}(\infty) f_{k}^{-1}$ assuming $f_{N+1}=f_{1}$. For $N=3$ it can be sketched as follows:

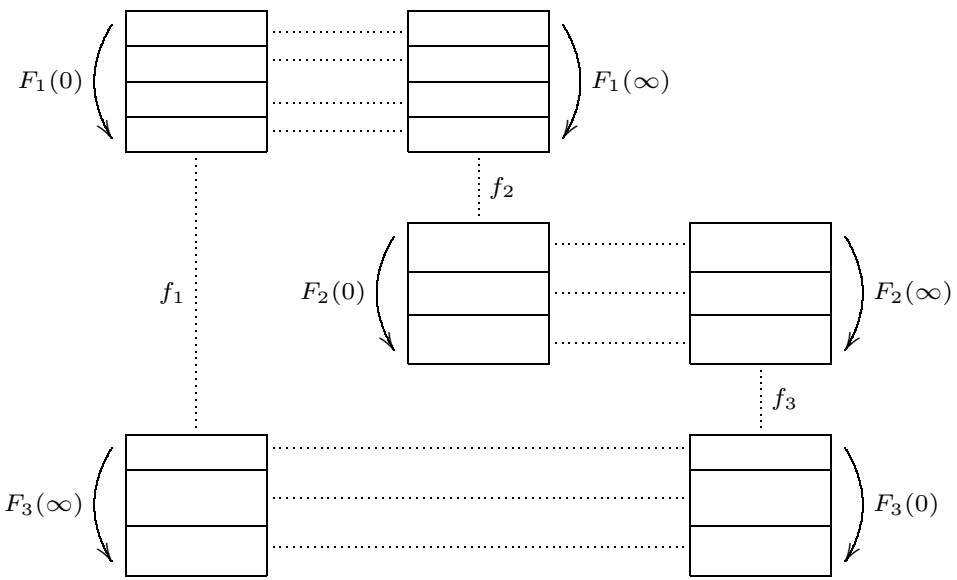

Since the matrices $F_{k}(0)$ and $F_{k}(\infty)$ have the block-triangular structure, as described above, thus the matrices $\mu_{k}(0)$ and $\mu_{k}(\infty)$ split into horizontal blocks labeled by $n \in \mathbb{Z}$, as in the decomposition of $\widetilde{\mathcal{F}}$. Such blocks contain $r(n, k)$ rows, and can be transformed only together by $F_{k}^{n n}(0)=F_{k}^{n n}(\infty)$. We call them conjugated blocks and connect them by dotted lines.

These types of matrix problems are well known in representation theory. They are called Gelfand problems or representations of bunches of chains (see GP68, Bon92). For an application of Gelfand problems to the classification of torsion-free sheaves on cycles of projective lines, we refer to DG01 (see also BBDG]).

\section{MATRIX PROBLEM FOR KodAIRA FIBERS II, III AND IV}

In this section we formulate the matrix problem $\mathrm{MP}_{E}$ for the other curves from Table 1. Let $E$ be a Kodaira fiber with $N(N \leq 3)$ components, $s$ the unique singular point and $\pi: \widetilde{E} \rightarrow E$ the normalization map. For example, for $N=3$ we have

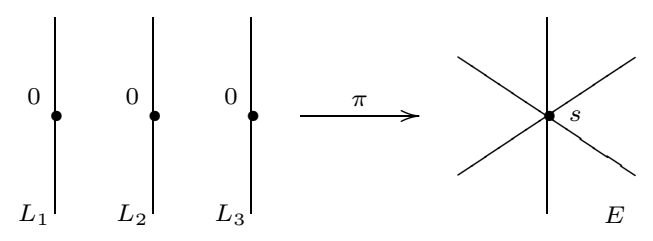

Note that $\widetilde{E}$ consists of a disjoint union of $N$ projective lines. On each component $L_{k}$ choose coordinates $\left(z_{0}: z_{1}\right)$ such that the preimage of the singular point $s=$ $(0: 0: 1)$ on $L_{k}$ is $0:=(0: 1)$. Let $U_{k}=\left\{\left(z_{0}: z_{1}\right) \mid z_{1} \neq 0\right\}$ be affine neighborhoods of 0 on $L_{k}$ with local coordinates $t_{k}:=z_{0} / z_{1}$ for $k=1, \ldots, N$, and let $U$ be the 
union $\bigcup_{k=1}^{N} \pi\left(U_{k}\right)$. Calculate the normalization map $\mathcal{O} \hookrightarrow \pi_{*} \widetilde{\mathcal{O}}=\pi_{*}\left(\bigoplus_{k=1}^{N} \mathcal{O}_{L_{k}}\right)$, the conductor $\mathcal{J}$, and the structure sheaves $\mathcal{O}_{S}$ and $\mathcal{O}_{\widetilde{S}}$ for each Kodaira fiber:

II. Let $E$ be a cuspidal cubic curve in $\mathbb{P}^{2}$ given by the equation $x^{3}-y^{2} z=$ 0 . Then locally the normalization map is $\mathbb{k}[U]=\mathbb{k}\left[t^{2}, t^{3}\right] \hookrightarrow \mathbb{k}[t]$. Since on $\pi(U)$ the conductor is $\mathcal{J}=\left\langle t^{2}, t^{3}\right\rangle$, we have $\mathcal{O}_{S} \cong \mathbb{k}(s)$ and $\mathcal{O}_{\widetilde{S}} \cong$ $\left(\mathbb{k}[\varepsilon] / \varepsilon^{2}\right)(0)$.

III. Let $E$ be a tacnode curve given by the equation $y\left(z y-x^{2}\right)=0$. Then the normalization map is $\mathbb{k}[U] \hookrightarrow \mathbb{k}\left[t_{1}\right] \oplus \mathbb{k}\left[t_{2}\right]$ taking $1 \mapsto(1,1), x \mapsto\left(t_{1}, t_{2}\right)$, and $y \mapsto\left(0, t_{2}^{2}\right)$. On $\pi(U)$ for the conductor we have $\mathcal{J}=\left\langle\left(t_{1}^{2}, 0\right),\left(0, t_{2}^{2}\right)\right\rangle$. In other words, the ideal sheaf of the scheme-theoretic preimage of $s$ is $\widetilde{\mathcal{J}}=\left(\mathcal{I}_{L_{1}, 0}^{2}, \mathcal{I}_{L_{2}, 0}^{2}\right)$, where $\mathcal{I}_{L_{k}, 0}$ denotes the ideal sheaf of the point 0 on the component $L_{k}$. Hence, $\mathcal{O}_{\widetilde{S}} \cong \widetilde{\mathcal{O}} / \widetilde{\mathcal{J}}=\mathcal{O}_{L_{1}} / \mathcal{I}_{L_{1}, 0}^{2} \oplus \mathcal{O}_{L_{2}} / \mathcal{I}_{L_{2}, 0}^{2}$. Altogether we get $\mathcal{O}_{S} \cong\left(\mathbb{k}[\varepsilon] / \varepsilon^{2}\right)(s)$, and $\mathcal{O}_{\widetilde{S}} \cong\left(\mathbb{k}\left[\varepsilon_{1}\right] / \varepsilon_{1}^{2}\right)(0) \oplus\left(\mathbb{k}\left[\varepsilon_{2}\right] / \varepsilon_{2}^{2}\right)(0)$ and the induced map $\mathcal{O}_{S} \hookrightarrow \tilde{\pi}_{*} \mathcal{O}_{\widetilde{S}}$ takes $\varepsilon$ to $\left(\varepsilon_{1}, \varepsilon_{2}\right)$.

IV. Let $E$ be a curve consisting of three concurrent projective lines in $\mathbb{P}^{2}$, given by the equation $x y(x-y)=0$. Then the normalization map is $\mathbb{k}[U] \hookrightarrow$ $\mathbb{k}\left[t_{1}\right] \oplus \mathbb{k}\left[t_{2}\right] \oplus \mathbb{k}\left[t_{3}\right]$, sending $1 \mapsto(1,1,1), x \mapsto\left(t_{1}, t_{2}, 0\right)$, and $y \mapsto\left(t_{1}, 0, t_{3}\right)$. Since $\mathcal{J}(U)=\left\langle x^{2}, y^{2}, x y\right\rangle$, we have $\mathcal{O}_{S}=\mathbb{k}[x, y] /\left\langle x^{2}, y^{2}, x y\right\rangle$. Note that the ideal sheaf $\widetilde{\mathcal{J}}:=\pi^{*} \mathcal{J}$ is locally generated by $\left(t_{1}^{2}, 0,0\right),\left(0, t_{2}^{2}, 0\right)$ and $\left(0,0, t_{3}^{2}\right)$; i.e. $\widetilde{\mathcal{J}}=\left(\mathcal{I}_{L_{1}, 0}^{2}, \mathcal{I}_{L_{2}, 0}^{2}, \mathcal{I}_{L_{3}, 0}^{2}\right)$, where $\mathcal{I}_{L_{k}, 0}$ is as above. Hence, $\mathcal{O}_{\widetilde{S}} \cong \bigoplus_{k=1}^{3} \mathcal{O}_{L_{k}} / \mathcal{I}_{L_{k}, 0}^{2}$.

Matrix problems $\mathrm{MP}_{E}$ for Kodaira fibers II, III and IV. For a triple $(\widetilde{\mathcal{F}}, \mathcal{M}, \tilde{\mu})$ we fix:

- a splitting $\tilde{\mathcal{F}} \cong \bigoplus_{k=1}^{N}\left(\bigoplus_{n \in \mathbb{Z}} \mathcal{O}_{L_{k}}(n)^{r(n, k)}\right)$ with $\sum_{n \in \mathbb{Z}} r(n, k)=r$;

- an isomorphism $\mathcal{M} \cong \mathcal{O}_{S}^{r}$;

- for each component $L:=L_{k}$ we take the trivializations

$$
\begin{aligned}
\mathcal{O}_{L}(n) \otimes \mathcal{O}_{L} / \mathcal{I}_{L, 0}^{2} & \longrightarrow \mathbb{k}\left[\varepsilon_{k}\right] / \varepsilon_{k}^{2}, \\
\zeta \otimes 1 & \longmapsto \operatorname{pr}\left(\frac{\zeta}{z_{1}^{n}}\right)
\end{aligned}
$$

for a local section $\zeta$ of $\mathcal{O}_{L_{k}}(n)$ on the open set $U_{k}$, where the projection

$$
p r: \mathbb{k}\left[U_{k}\right] \longrightarrow \mathbb{k}\left[\varepsilon_{k}\right] / \varepsilon_{k}^{2}
$$

is the map induced by $\mathbb{k}\left[t_{k}\right] \longrightarrow \mathbb{k}\left[\varepsilon_{k}\right] / \varepsilon_{k}^{2}$, mapping $t_{k} \mapsto \varepsilon_{k}$.

With respect to all these choices we have:

- The map $\tilde{\mu}$ can be written as a combination of $2 N r \times r$ matrices over $\mathbb{k}$ :

$$
\tilde{\mu}=\left(\mu_{1}, \ldots, \mu_{N}\right)=\left(\mu_{1}(0)+\varepsilon_{1} \cdot \mu_{\varepsilon_{1}}(0), \ldots, \mu_{N}(0)+\varepsilon_{N} \cdot \mu_{\varepsilon_{N}}(0)\right) .
$$

The morphism $\tilde{\mu}$ is invertible if and only if all $\mu_{k}(0) \in \mathrm{GL}(\mathbb{k}, r)$.

- If on a component $L=L_{k}$ we have a morphism $\mathcal{O}_{L}(n) \rightarrow \mathcal{O}_{L}(m)$ given by a homogeneous form $Q\left(z_{0}, z_{1}\right)$ of degree $m-n$, then the induced map 
$\mathcal{O}_{L}(n) \otimes \mathcal{O}_{\widetilde{S}} \longrightarrow \mathcal{O}_{L}(m) \otimes \mathcal{O}_{\widetilde{S}}$ is given by the map

$$
\operatorname{pr}\left(Q\left(z_{0}, z_{1}\right) / z_{1}^{m-n}\right)=Q(0: 1)+\varepsilon_{k} \frac{d Q}{d z_{0}}(0: 1) .
$$

Hence, for a morphism $(F, f):(\widetilde{\mathcal{F}}, \mathcal{M}, \tilde{\mu}) \longrightarrow\left(\widetilde{\mathcal{F}}^{\prime}, \mathcal{M}^{\prime}, \tilde{\mu}^{\prime}\right)$ the induced map $\tilde{\imath}^{*} F: \tilde{\imath}^{*} \widetilde{\mathcal{F}} \longrightarrow \tilde{\imath}^{*} \widetilde{\mathcal{F}}^{\prime}$ is

$$
\left.\tilde{\imath}^{*} F\right|_{L}=F_{k}(0)+\varepsilon_{k} \frac{d F_{k}}{d z_{0}}(0) \in \operatorname{Mat}\left(\mathbb{k}\left[\varepsilon_{k}\right] / \varepsilon_{k}^{2}, r\right),
$$

where, as usual, $F_{k}(0)$ denotes $F_{k}(0: 1)$.

- The morphism $\tilde{\pi}^{*} f$ consists of $N$ copies of the matrix $f$, where

- $f \in \operatorname{Mat}(\mathbb{k}, r \times r)$ for the cuspidal cubic;

- $f=f(0)+f_{\varepsilon}(0) \in \operatorname{Mat}\left(\mathbb{k}[\varepsilon] / \varepsilon^{2}, r \times r\right)$, for $\varepsilon=\left(\varepsilon_{1}, \varepsilon_{2}\right)$ for the tacnode curve (Kodaira fiber III);

- $f=f(0)+x \cdot f_{x}(0)+y \cdot f_{y}(0) \in \operatorname{Mat}\left(\mathbb{k}[x, y] /\left\langle x^{2}, y^{2}, x y\right\rangle, r \times r\right)$ for the three lines through a point in a plane (Kodaira fiber IV).

A morphism $(F, f)$ is an automorphism if and only if all $F_{k}(0)$ for $k \in\{1, \ldots, N\}$ and $f(0)$ are invertible $r \times r$ matrices over $\mathbb{k}$. For example, for the Kodaira fiber IV we get the following matrix problem. There are six $r \times r$ matrices $\mu_{1}(0)$, $\mu_{\varepsilon_{1}}(0), \mu_{2}(0), \mu_{\varepsilon_{2}}(0)$ and $\mu_{3}(0), \mu_{\varepsilon_{3}}(0)$, where all $\mu_{k}(0)$ are invertible. The pairs $\mu_{k}(0), \mu_{\varepsilon_{k}}(0)$ are simultaneously divided into horizontal blocks with respect to the splitting of $\left.\widetilde{\mathcal{F}}\right|_{L_{k}}$.

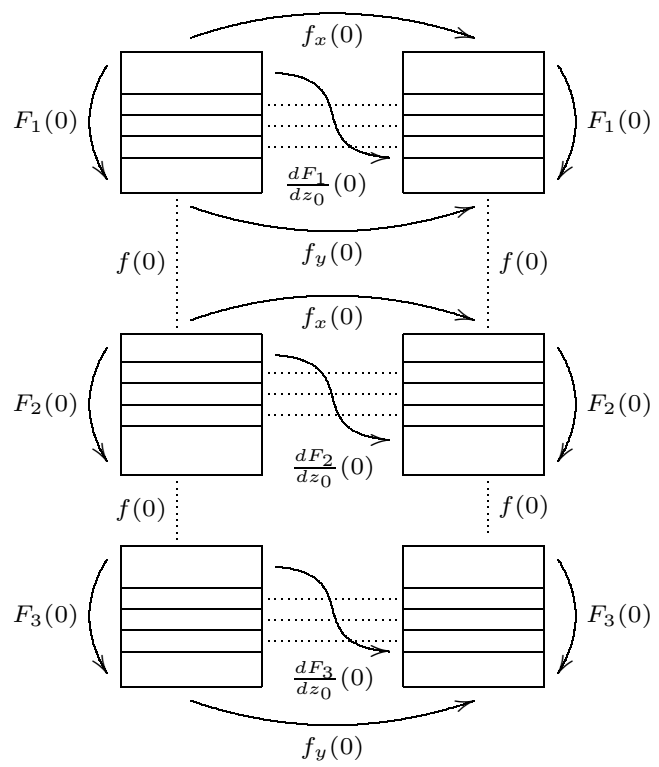

Note that $f_{x}(0)$ does not act on $\mu_{\varepsilon_{3}}$ and $f_{y}(0)$ does not act on $\mu_{\varepsilon_{2}}$ since, as explained above, the normalization map $\mathcal{O}_{S} \hookrightarrow \tilde{\pi}_{*} \mathcal{O}_{\widetilde{S}}$ sends $x \mapsto\left(\varepsilon_{1}, \varepsilon_{2}, 0\right)$, and $y \mapsto\left(\varepsilon_{1}, 0, \varepsilon_{3}\right)$.

If we restrict this problem on the first two components and assume $f_{y}(0)=0$ and $f_{\varepsilon}:=f_{x}(0)$ we obtain the matrix problem for a tacnode curve. If we restrict it to the first component with $f_{y}(0)=f_{x}(0)=0$ we get the matrix problem for the cuspidal cubic curve. Each of this problems is wild even for two horizontal 
blocks; see [Dro92, Section 1] or [BD09. However, the simplicity condition of a triple $(\widetilde{\mathcal{F}}, \mathcal{M}, \tilde{\mu})$ imposes some additional restrictions making the problem tame.

\section{Simplicity CONDition}

A vector bundle on a curve $X$ is called simple if it admits no endomorphisms but homotheties; i.e., $\operatorname{End}_{X}(\mathcal{F})=\mathbb{k}$ and the subcategory of simple vector bundles is denoted by $\mathrm{VB}_{X}^{s}$. This notion can be obviously translated to the language of triples. In terms of matrix problems: an object $\tilde{\mu}$ of $\mathrm{MP}_{X}$ is called a brick if $\operatorname{End}_{\mathrm{MP}_{X}}(\tilde{\mu})=\mathbb{k}$. The full subcategory of bricks is denoted by $\mathrm{MP}_{X}^{s}$ and $\mathrm{MP}_{X}^{s}(\mathbb{r})$ if the dimension vector $\mathbb{r}$ is fixed. Note that a nonscalar morphism $(F, f)$ can have a scalar restriction $\left(\tilde{\imath}^{*} F, \tilde{\pi}^{*} f\right)$.

Lemma 5.1. Let $X$ be a rational singular curve and $(\widetilde{\mathcal{F}}, \mathcal{M}, \tilde{\mu}) \in \operatorname{Tr}_{X}$ be a triple. Then the map $\operatorname{End}_{\operatorname{Tr}_{X}}(\widetilde{\mathcal{F}}, \mathcal{M}, \tilde{\mu}) \rightarrow \operatorname{End}_{\mathrm{MP}_{X}}(\tilde{\mu})$ is bijective if and only if for all the components $L$ of $\widetilde{X}$ and for all summands $\mathcal{O}_{L}(n) \oplus \mathcal{O}_{L}(m)$ of $\left.\widetilde{\mathcal{F}}\right|_{L}$ the canonical maps $\operatorname{Hom}\left(\mathcal{O}_{L}(n), \mathcal{O}_{L}(m)\right) \rightarrow \mathbb{k}[\widetilde{S} \cap L]$, taking $Q \mapsto \tilde{\imath}^{*} Q$, are bijective.

This obvious lemma implies certain nice properties for a matrix problem under the simplicity condition. For the curves under consideration, we have the following:

Lemma 5.2. Let $E$ be a Kodaira fiber $I_{N}$, (for $N \in \mathbb{N}$ ) II, III or $I V$, and let $(\widetilde{\mathcal{F}}, \mathcal{M}, \tilde{\mu}) \in \operatorname{Tr}_{X}$ be a simple triple, i.e. $\operatorname{End}_{\operatorname{Tr}_{X}}(\widetilde{\mathcal{F}}, \mathcal{M}, \tilde{\mu})=\mathbb{k}$. Then for each component $L:=L_{k}(1 \leq k \leq N)$,

$$
\left.\widetilde{\mathcal{F}}\right|_{L}=\left(\mathcal{O}_{L}\left(n_{k}\right)\right)^{r-\bar{d}_{k}} \oplus\left(\mathcal{O}_{L}\left(n_{k}+1\right)\right)^{\bar{d}_{k}}
$$

for some $n_{k} \in \mathbb{Z}$ and $1 \leq \bar{d}_{k} \leq r$.

Proof. Assume that $\left.\pi^{*} \mathcal{F}\right|_{L}$ contains a summand $\mathcal{O}_{L}(n) \oplus \mathcal{O}_{L}(m)$ with $m \geq n+2$. Let $\left(z_{0}: z_{1}\right)$ be the local coordinates as in Sections 3 and 4 . Since the degree $m-n \geq 2$, there exists a nonzero homogeneous form $Q \in \operatorname{Hom}_{L}\left(\mathcal{O}_{L}(n), \mathcal{O}_{L}(m)\right) \cong \mathbb{k}\left[z_{0}, z_{1}\right]_{m-n}$ such that $\tilde{\imath}^{*} Q=0$. Indeed, if $E$ is a Kodaira cycle, then $\tilde{\imath}^{*} Q=(Q(0), Q(\infty))$ and if $E$ is a Kodaira fiber of type II, III or IV, then the restriction of $\widetilde{\mathcal{J}}$ to the component $L$ is $\mathcal{I}_{L, 0}^{2} \subset \mathcal{O}_{L, 0}$ and thus $\tilde{\imath}^{*} Q=Q(0)+\frac{\partial Q}{\partial z_{0}}(0)$. In both cases the map $Q \mapsto \tilde{\imath}^{*} Q$ is not injective and we get a contradiction to the condition of Lemma 5.1

Remark 5.3. Note that the twists $n_{k}$ do not affect the matrix problem. Hence we can assume that the blocks have weights 0 and 1 for each component $L_{k}$ and replace the multidegree $\mathbb{d}$ by $\left(\bar{d}_{1}, \ldots, \bar{d}_{N}\right)$ and the degree $d$ by $\bar{d}:=\bar{d}_{1}+\cdots+\bar{d}_{N}$, where $\bar{d}_{k}=d_{k} \bmod r$. Having the twists $n_{k}$ we can recover the multidegree of $\mathbb{d}$ by the rule $d_{k}=r \cdot n_{k}+\bar{d}_{k}$.

\section{Primary Reduction}

Applying condition (5.1) to the matrix problem $\mathrm{MP}_{E}$ we obtain that each matrix consists of at most two horizontal blocks. Despite this simplification the problem remains quite cumbersome. However, it can be reduced to a partial canonical form, such that all its matrices but one consist of the identity and zero blocks. We denote by $M$ the remaining nonreduced matrix and formulate for it a new matrix problem. 
It seems reasonable to introduce some simplified system of notation.

- Let $\mathbb{1}$ denotes the identity blocks, 0 the zero blocks,

- use the star * to denote nonreduced blocks and small Latin letters for a finer specification.

The matrix $M$ is divided into blocks, the set of column-blocks coincides with the set of row-blocks and is denoted by $I=\{1,2, \ldots,|I|\}$. Then $\mathbb{s}=\left(s_{1}, \ldots, s_{|I|}\right) \in \mathbb{N}^{I}$ is the dimension vector of $M$.

6.1. Nodal cubic curve. According to Section 3 the matrix problem $\mathrm{MP}_{E}$ for the nodal cubic curve $E$ with two blocks is as follows:

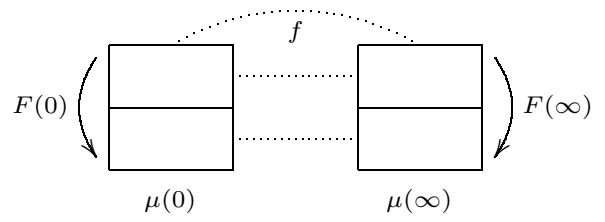

Since the normalization consists of a unique component $L$ we skip the indices by $F, f$ and $\mu$. As was mentioned above, both matrices $\mu(0)$ and $\mu(\infty)$ are invertible. We reduce one of them, say $\mu(0)$, to the identity form:

$$
\mu(0)=\begin{array}{|l|l|}
\hline \mathbb{1} & 0 \\
\hline 0 & \mathbb{1} \\
\hline
\end{array} \quad \text { and } M:=\mu(\infty)=\begin{array}{|c|c|}
\hline a_{1} & b \\
\hline c & a_{2} \\
\hline
\end{array}
$$

To preserve $\mu(0)$ unchanged we assume $f=F(0)$. Reformulate the problem for the matrix $M:=\mu(\infty)$. The transformation rule is $M \mapsto S M\left(S^{\prime}\right)^{-1}$, where

$$
\left(S, S^{\prime}\right):=(F(\infty), F(0))=\left(\begin{array}{|c|c||c|c|}
\hline w_{1} & 0 & w_{1} & 0 \\
\hline u & w_{2} & v & w_{2} \\
\hline
\end{array}\right.
$$

Note that the sizes of the blocks are determined by rank and degree: $\left(s_{1}, s_{2}\right)=$ $(r-\bar{d}, \bar{d})$, where $\bar{d}:=d \bmod r$.

6.2. Cuspidal cubic curve. Recall the problem $\mathrm{MP}_{E}$ on two blocks for the cuspidal curve:

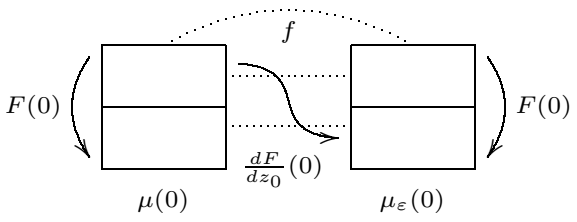

As in the case of a nodal curve we skip the indices by $F, f$ and $\mu$. The matrix $\mu(0)$ can be reduced to the identity form. To preserve this form unchanged we assume $F(0)=f$. Moreover, using the transformations $\frac{d F}{d z_{0}}(0)$, we can obtain zeros on the left lower block of $\mu_{\varepsilon}(0)$ :

$$
\mu(0)=\begin{array}{|l|l|}
\hline \mathbb{1} & 0 \\
\hline 0 & \mathbb{1} \\
\hline
\end{array} \quad \text { and } M:=\mu_{\varepsilon}(0)=\begin{array}{|c|c|}
\hline a_{1} & b \\
\hline 0 & a_{2} \\
\hline
\end{array} .
$$


We obtain a new matrix problem which reads: $M \mapsto S M S^{-1} \bmod \left(\begin{array}{ll}0 & 0 \\ \times & 0\end{array}\right)$, where the matrix $S$ inherits the same lower-block-triangular structure as $F(0)$ :

$$
S:=F(0)=f=\begin{array}{|c|c|}
\hline w_{1} & 0 \\
\hline u & w_{2} \\
\hline
\end{array} .
$$

As in the previous case, the sizes of blocks are determined by rank and degree:

$$
\left(s_{1}, s_{2}\right)=(r-\bar{d}, \bar{d}) \text {, where } \bar{d}:=d \bmod r .
$$

6.3. Cycle of two lines. According to Section 3 the original matrix problem $\mathrm{MP}_{E}$ for a cycle of two lines with two blocks on each component is

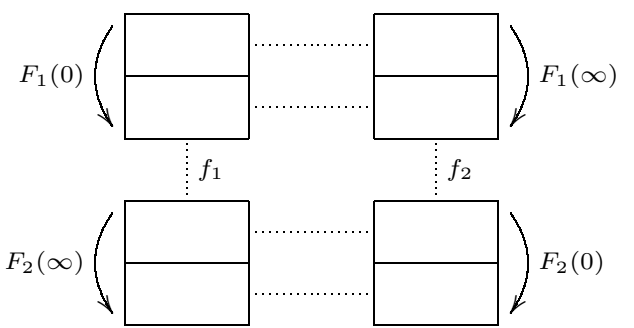

All four matrices $\left(\mu_{1}(0), \mu_{1}(\infty), \mu_{2}(\infty), \mu_{2}(0)\right)$ are invertible. Two diagonal matrices, say $\mu_{1}(0)$ and $\mu_{2}(0)$, can be reduced to the identity form. Then one of the others, say $\mu_{2}(\infty)$, can be reduced to the form:

\begin{tabular}{|ll|ll|l|}
\hline & 2 & 3 & 4 & \\
$\mathbb{1}$ & 0 & 0 & 0 & 1 \\
0 & 0 & $\mathbb{1}$ & 0 & 3 \\
\hline 0 & $\mathbb{1}$ & 0 & 0 & 2 \\
0 & 0 & 0 & $\mathbb{1}$ & 4 \\
\hline
\end{tabular}

Transformations $(F, f)$ preserving the reduced matrices $\mu_{1}(0), \mu_{2}(0)$ and $\mu_{2}(\infty)$ unchanged satisfy the equations

$$
f_{1}=F_{1}(0), f_{2}=F_{2}(0) \text { and } F_{2}(\infty) \mu_{2}(\infty)=\mu_{2}(\infty) f_{1} .
$$

This implies the following triangular structures for $F_{1}(0)$ and $F_{2}(\infty)$ :

$$
F_{1}(0)=\begin{array}{|cc|cc}
w_{1} & 0 & 0 & 0 \\
x_{21} & w_{2} & 0 & 0 \\
\hline x_{31} & 0 & w_{3} & 0 \\
x_{41} & x_{42} & x_{43} & w_{4}
\end{array} \quad \text { and } F_{2}(\infty)=\begin{array}{|ll|ll|}
w_{1} & 0 & 0 & 0 \\
x_{31} & w_{3} & 0 & 0 \\
\hline x_{21} & 0 & w_{2} & 0 \\
x_{41} & x_{43} & x_{42} & w_{4} \\
\hline
\end{array}
$$

Since the diagonal blocks of $F_{k}(0)$ and $F_{k}(\infty)$ coincide (for $k=1,2$ ), we also have:

$$
F_{1}(\infty)=\begin{array}{|cc|cc}
\hline w_{1} & 0 & 0 & 0 \\
x_{21} & w_{2} & 0 & 0 \\
\hline y_{31} & y_{32} & w_{3} & 0 \\
y_{41} & y_{42} & x_{43} & w_{4}
\end{array} \quad \text { and } F_{2}(0)=\begin{array}{|cc|cc|}
w_{1} & 0 & 0 & 0 \\
x_{31} & w_{3} & 0 & 0 \\
\hline z_{21} & z_{23} & w_{2} & 0 \\
z_{41} & z_{43} & x_{42} & w_{4} \\
\hline
\end{array}
$$


Reduced matrix problem. Thus we obtain a new problem for the matrix $M:=$ $\mu_{1}(\infty)$ with the transformations $M \mapsto S M\left(S^{\prime}\right)^{-1}$, where $\left(S, S^{\prime}\right):=\left(F_{1}(\infty), F_{2}(0)\right)$. Note that if the sizes of blocks 1 and 4 are both nonzero, then taking a nonzero entry $x_{41}$ of the matrices $F_{2}(\infty)$ and $F_{1}(0)$ we obtain a nonscalar endomorphism. Hence, there are no sincere bricks and the maximal tuples of blocks are $I=(1,2,3)$ and its dual $I=(2,3,4)$. The dimension vector $\mathbb{s}=\left(s_{i}\right)_{i \in I}$ and the matrix problem are determined by $r$ and $\left(\bar{d}_{1}, \bar{d}_{2}\right)$, where $\bar{d}_{k}=d_{k} \bmod r$ and $\bar{d}=\bar{d}_{1}+\bar{d}_{2}$, as shown in Table 2,

TABLE 2

\begin{tabular}{|c|c|c|c|c|}
\hline & condition & set $I$ & dimension vector $\$$ & state \\
\hline \hline 1. & $r \geq \bar{d}$ & $(1,2,3)$ & $\left(r-\bar{d}, \bar{d}_{2}, \bar{d}_{1}\right)$ & $A^{+}$ \\
\hline $1^{\prime}$. & $r<\bar{d}$ & $(2,3,4)$ & $\left(r-\bar{d}_{1}, r-\bar{d}_{2}, \bar{d}-r\right)$ & $A^{-}$ \\
\hline
\end{tabular}

Here $A^{+}$denotes the problem $M \mapsto S M\left(S^{\prime}\right)^{-1}$, on the set of blocks $I=\left\{i_{1}, i_{2}, i_{3}\right\}$ with

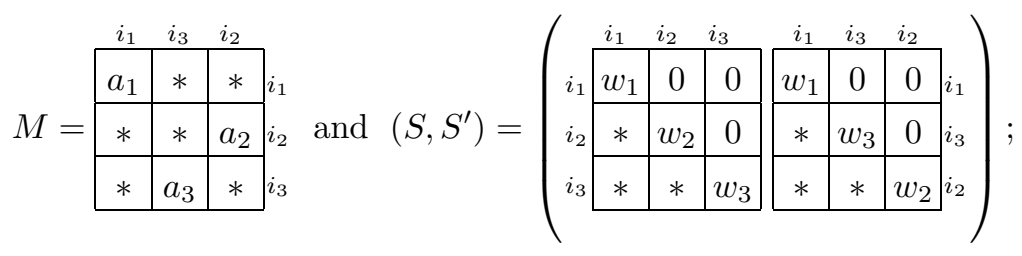

the problem $A^{-}$is $M \mapsto S M\left(S^{\prime}\right)^{-1}$, on the set of vertices $I=\left\{i_{1}, i_{2}, i_{3}\right\}$, where

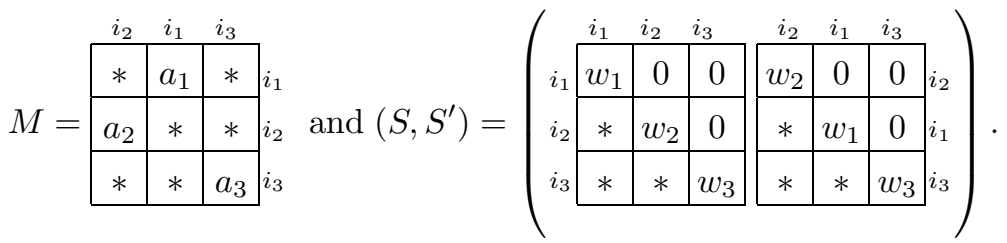

Note that since matrices $S$ and $S^{\prime}$ are lower triangular, both problems $A^{+}$or $A^{-}$ can be recognized by the form of the matrix $M$.

6.4. Tacnode curve. Analogously as in the previous case, we reduce the matrix $\mu_{1}(0)$ to the identity form and the matrix $\mu_{2}(0)$ to the form (6.1). Then for the transformations we have the restrictions:

$$
f(0)=F_{1}(0) \text { and } F_{2}(0) \mu_{2}(0)=\mu_{2}(0) f(0),
$$

and consequently $F_{1}(0)$ is as in (6.3). By the transformation $f_{\varepsilon}$ we can reduce one of the matrices, either $\mu_{\varepsilon_{1}}(0)$ or $\mu_{\varepsilon_{2}}(0)$, say $\mu_{\varepsilon_{2}}(0)$, to the zero form. In the remaining matrix $M:=\mu_{\varepsilon_{1}}(0)$, the blocks (31), (32), (41) and (42) can be reduced to zero by the transformation $\frac{d F_{k}}{d z_{0}}(0)$, and the blocks $(21),(23),(41)$ and $(43)$ can be killed by $f_{\varepsilon}$. 
Reduced matrix problem. Thus we obtain a new problem for the matrix $M$ with the transformations $M \mapsto S M S^{-1}$ modulo the zero-block entries of $M$ :

$$
\left.M:=\mu_{\varepsilon_{1}}(0)=\begin{array}{cc|cc}
a_{1} & b_{12} & b_{13} & b_{14} \\
0 & a_{2} & 0 & b_{24} \\
\hline 0 & 0 & a_{3} & b_{34} \\
0 & 0 & 0 & a_{4}
\end{array}\right] \text { and } S:=F_{1}(0)=\begin{array}{|cc|cc|}
w_{1} & 0 & 0 & 0 \\
x_{21} & w_{2} & 0 & 0 \\
\hline x_{31} & 0 & w_{3} & 0 \\
x_{41} & x_{42} & x_{43} & w_{4} \\
\hline
\end{array} \text {. }
$$

It is easy to see that if the sizes of blocks 1 and 4 are both nonzero, then there is a nontrivial endomorphism. As in the previous case there are no sincere bricks and the admissible tuples of blocks $I$ and sizes $\$$ are the same as in Table 2 , whereas the configurations $A^{+}$and $A^{-}$are respectively the matrix problems with

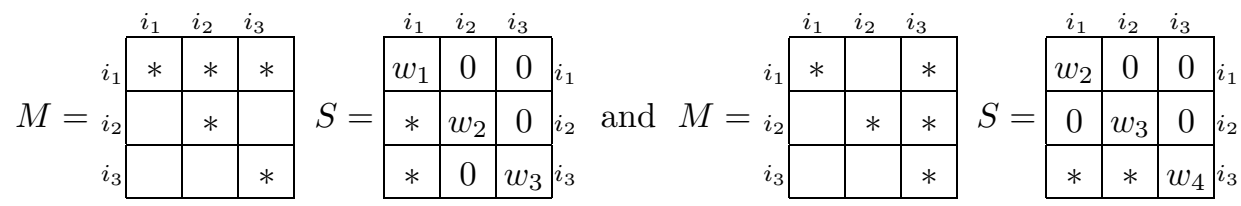

In the matrix $M$, we replaced the zero-blocks by the empty spaces, since they do not play any role in the calculations and can always be restored for some outer reasons.

Example 6.1. Let $E$ be a Kodaira fiber $\mathrm{I}_{2}$ or III and $(\widetilde{\mathcal{F}}, \mathcal{M}, \tilde{\mu})$ be a triple corresponding to a simple vector bundle. If $r \geq \bar{d}$, then the matrix $\tilde{\mu}$ can be respectively transformed to the form

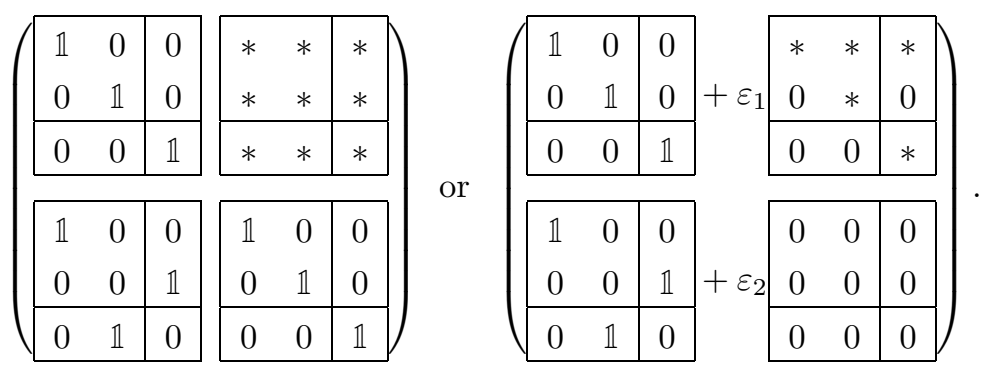


6.5. Cycle of tree lines. According to Section 3 the original matrix problem $\mathrm{MP}_{E}$ with two blocks on each component is

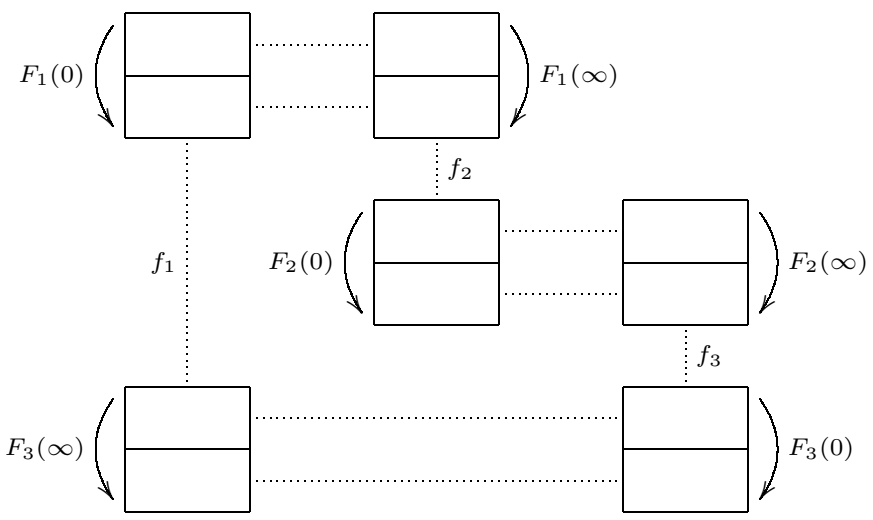

Matrices $\mu_{1}(0), \mu_{2}(0)$ and $\mu_{3}(0)$ can be reduced to the identity form. The matrix $\mu_{3}(\infty)$ can be reduced to the form (6.1). For the morphisms we have

$$
f_{1}=F_{1}(0), f_{2}=F_{2}(0), f_{3}=F_{3}(0) \text { and } F_{3}(\infty) \mu_{3}(\infty)=\mu_{3}(\infty) f_{1}
$$

Then the matrix $f_{3}$ becomes a special block-triangular structure. In other words, the matrix $\mu_{2}(\infty)$ is subdivided into four column-blocks: a column can be added to any other column from a block on the left and it cannot be added to a column from another block on the right. Thus $\mu_{2}(\infty)$ can be reduced to the form

$\mu_{2}(\infty)=$\begin{tabular}{lllllllllll|l}
1 & 2 & 5 & 6 & 3 & 4 & 7 & 8 & \\
$\mathbb{1}$ & 0 & 0 & 0 & 0 & 0 & 0 & 0 & 1 \\
0 & 0 & $\mathbb{1}$ & 0 & 0 & 0 & 0 & 0 & 5 \\
0 & 0 & 0 & 0 & $\mathbb{1}$ & 0 & 0 & 0 & 3 \\
0 & 0 & 0 & 0 & 0 & 0 & $\mathbb{1}$ & 0 & 7 \\
\hline 0 & $\mathbb{1}$ & 0 & 0 & 0 & 0 & 0 & 0 & 2 \\
0 & 0 & 0 & $\mathbb{1}$ & 0 & 0 & 0 & 0 & 6 \\
0 & 0 & 0 & 0 & 0 & $\mathbb{1}$ & 0 & 0 & 4 \\
0 & 0 & 0 & 0 & 0 & 0 & 0 & $\mathbb{1}$ & 8 \\
0
\end{tabular}

Reduced matrix problem. The remaining nonreduced matrix is $M:=\mu_{1}(\infty)$. For it we obtain the problem $M \mapsto S M\left(S^{\prime}\right)^{-1}$, where the transformations are $\left(S, S^{\prime}\right)=\left(F_{1}(\infty), f_{2}\right)$. Equations (6.5) together with $F_{2}(\infty) \mu_{2}(\infty)=\mu_{2}(\infty) f_{3}$ imply the triangular forms for the matrices $F_{k}(0), F_{k}(\infty)$ and $f_{k}$ (for $k=1,2,3$ ); in 
particular:

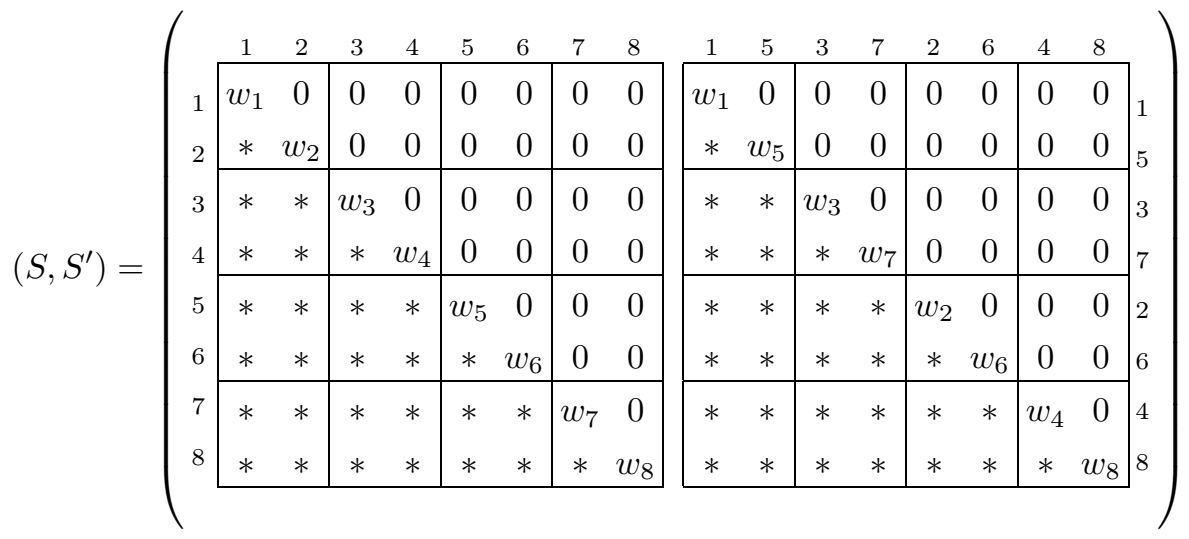

The stars $*$ denote arbitrary blocks and $w_{i}$ for $i \in\{1, \ldots, 8\}$ are the common diagonal blocks. The transformations of row and column-blocks of $M$ are clear: a row can be added to any other row from a block below and it cannot be added to a row from a block above it; and a column can be added to any other column from a block on the left and it cannot be added to a column from a block on the right.

Nontrivial endomorphisms. Analogously as in the case of a cycle of two lines there are some pairs $(i, j) \in I \times I$ such that if $s_{i} \cdot s_{j}>0$, then there exists a nontrivial endomorphism. Such blocks are called mutually excluding and denoted by $i \cap j$.

- If the matrices $F_{3}(\infty)$ and $F_{1}(0)$ contain at least one of the following entries: (71), (81), (72) or (82), then there is a nontrivial endomorphism. In our short notation we have intersections $1,2 \cap 7,8$.

- Analogously we have $1,5 \cap 4,8$ coming from the matrices $F_{3}(0)$ and $F_{2}(\infty)$.

- The blocks 1 and 6 are mutually excluding; the endomorphism is induced by the entry $(61)$ of the matrices $F_{3}(0), F_{3}(\infty)$ and $F_{2}(\infty)$. Similarly, there is an endomorphism for the pair (38) induced by the matrices $F_{1}(0), F_{3}(\infty)$ and $F_{3}(0)$.

All the mutually excluding blocks can be indicated on the intersection diagram:

$$
2{ }^{2}{ }_{8}^{1}-54
$$

The diagram reads as follows: a matrix $M$ is a brick if it contains no pair of blocks $(i, j)$ such that $i$ and $j$ in the diagram are separated by $\cap$ and are either in the same column or one of them is 1 or 8 .

In the following table we present the maximal tuples of blocks $I=\left(i_{1}, i_{2}, i_{3}, i_{4}\right)$ for $M$ being a brick, express the dimension vector $\$=\left(s_{i_{1}}, s_{i_{2}}, s_{i_{3}}, s_{i_{4}}\right) \in \mathbb{N}^{4}$ in terms of rank and multidegree and, moreover, answer the question when such a tuple of blocks appears and specialize the matrix problems in each case.

The configurations $A^{+}, A^{-}$and $C$ on the set of blocks $I=\left\{i_{1}, i_{2}, i_{3}, i_{4}\right\}$ encode matrix problems $M \mapsto S M\left(S^{\prime}\right)^{-1}$, where $S$ and $S^{\prime}$ are block-triangular and the 
TABLE 3

\begin{tabular}{|r|c|c|c|c|}
\hline & condition & set $I$ & dimension vector $\$$ & state \\
\hline \hline 1. & $r \geq \bar{d}$ & $(1,2,3,5)$ & $\left(r-\bar{d}, \bar{d}_{2}, \bar{d}_{3}, \bar{d}_{1}\right)$ & $A^{+}$ \\
\hline $1^{\prime}$ & $\bar{d} \geq 2 r$ & $(4,6,7,8)$ & $\left(r-\bar{d}_{1}, r-\bar{d}_{3}, r-\bar{d}_{2}, \bar{d}-2 r\right)$ & $A^{-}$ \\
\hline \hline 2. & $\bar{d}>r>\left(\bar{d}_{2}+\bar{d}_{3}\right),\left(\bar{d}_{1}+\bar{d}_{3}\right)$ & $(2,3,5,6)$ & $\left(r-\left(\bar{d}_{1}+\bar{d}_{3}\right), \bar{d}_{3}, r-\left(\bar{d}_{2}+\bar{d}_{3}\right), \bar{d}-r\right)$ & $A^{-}$ \\
\hline $2^{\prime}$. & $\left(\bar{d}_{2}+\bar{d}_{3}\right),\left(\bar{d}_{1}+\bar{d}_{3}\right)>r$ & $(3,4,6,7)$ & $\left(2 r-\bar{d},\left(\bar{d}_{2}+\bar{d}_{3}\right)-r, r-\bar{d}_{3},\left(\bar{d}_{1}+\bar{d}_{3}\right)-r\right)$ & $A^{+}$ \\
\hline \hline 3. & $\left(\bar{d}_{2}+\bar{d}_{3}\right) \geq r \geq\left(\bar{d}_{1}+\bar{d}_{3}\right)$ & $(2,3,4,6)$ & $\left(r-\left(\bar{d}_{1}+\bar{d}_{3}\right), r-\bar{d}_{2},\left(\bar{d}_{2}+\bar{d}_{3}\right)-r, \bar{d}_{1}\right)$ & $C$ \\
\hline $3^{\prime}$. & $\left(\bar{d}_{1}+\bar{d}_{3}\right) \geq r \geq\left(\bar{d}_{2}+\bar{d}_{3}\right)$ & $(3,5,6,7)$ & $\left(r-\bar{d}_{1}, r-\left(\bar{d}_{2}+\bar{d}_{3}\right), \bar{d}_{2},\left(\bar{d}_{1}+\bar{d}_{3}\right)-r\right)$ & $C$ \\
\hline
\end{tabular}

matrix $M$ is defined as follows:

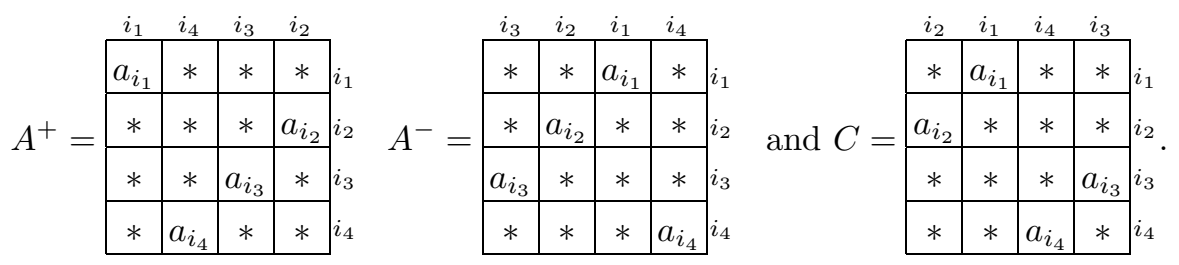

6.6. Three concurrent lines in a plane. Let $E$ be the Kodaira fiber IV and $\mathrm{MP}_{E}$ the matrix problem formulated in Section 4 with two blocks for each component. Reduce matrices $\mu_{1}(0)$ and $\mu_{2}(0)$ to the identity and (6.1) forms, respectively, as in the case of a tacnode curve. Then the transformations satisfy equations (6.4). Let us find a canonical form of $\mu_{3}(0)$ with respect to the transformations

$$
\mu_{3}(0) \mapsto F_{3}(0) \mu_{3}(0) f(0)^{-1} .
$$

The splitting of $F_{3}(0)$ and $f(0)$ into blocks induces the same column block structure for $\mu_{3}(0)$ as in the case of a cycle of three lines. However, on the contrary to that case, there is no addition from the third column-block to the second one. Thus proceeding as before instead of the form (6.6) we obtain only the following:

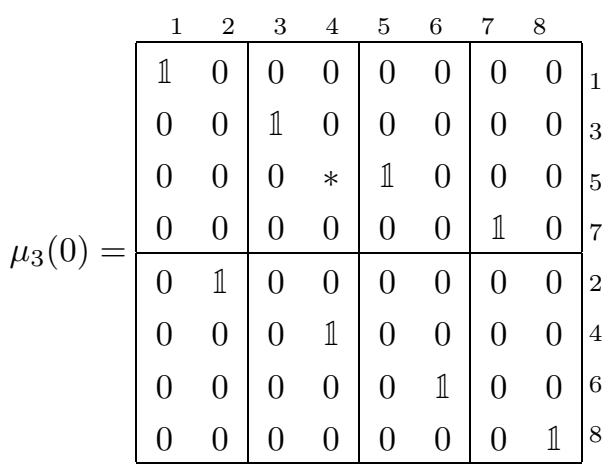

It turns out that the remaining block $*$ can be reduced to the form $\left(\begin{array}{ll}0 & 0 \\ 1 & 0\end{array}\right)$ as well. That implies subdivisions for the reduced blocks marked by 4 and 5 and a change 
of notation is required: $4 \mapsto(0,4)$ and $5 \mapsto(5,0)$. The equation $F_{3}(0) \mu_{3}(0)=$ $\mu_{3}(0) f(0)$ implies that the matrix $F_{1}(0)$ preserving $\mu_{3}(0)$ is as follows:

$F_{1}(0)=$\begin{tabular}{r|cc|cccccccc|}
1 & 2 & 3 & 0 & 4 & 5 & 0 & 6 & 7 & 8 \\
\hline & $w_{0}$ & 0 & 0 & 0 & 0 & 0 & 0 & 0 & 0 & 0 \\
2 & $*$ & $w_{1}$ & 0 & 0 & 0 & 0 & 0 & 0 & 0 & 0 \\
\hline 3 & $*$ & 0 & $w_{2}$ & 0 & 0 & 0 & 0 & 0 & 0 & 0 \\
0 & $*$ & $x$ & $*$ & $z$ & 0 & 0 & 0 & 0 & 0 & 0 \\
4 & $*$ & $*$ & $*$ & $*$ & $w_{4}$ & 0 & 0 & 0 & 0 & 0 \\
\cline { 2 - 9 } & $*$ & 0 & 0 & 0 & 0 & $w_{5}$ & 0 & 0 & 0 & 0 \\
0 & $*$ & $x$ & 0 & 0 & 0 & $*$ & $z$ & 0 & 0 & 0 \\
6 & $*$ & $*$ & 0 & 0 & 0 & $*$ & $*$ & $w_{7}$ & 0 & 0 \\
\hline & $*$ & 0 & $*$ & $y$ & 0 & $*$ & $y$ & 0 & $w_{8}$ & 0 \\
8 & $*$ & $*$ & $*$ & $*$ & $*$ & $*$ & $*$ & $*$ & $*$ & $w_{9}$ \\
\hline
\end{tabular}

As usual, the stars $*$ denote different blocks appearing only one time and $x, y$ and $z$ are some blocks appearing twice. By proper $f_{x}(0)$ and $f_{y}(0)$ the matrices $\mu_{\varepsilon_{2}}(0)$ and $\mu_{\varepsilon_{3}}(0)$ can be reduced to zero.

Reduced matrix problem. As usual, take $M:=\mu_{\varepsilon_{1}}(0)$ and transformations $M \mapsto S M S^{-1}$ modulo zero-blocks of $M$, where $S:=F_{1}(0)$. By proper $F_{1}(0), f_{x}(0)$ and $f_{y}(0)$ it can be reduced to the form

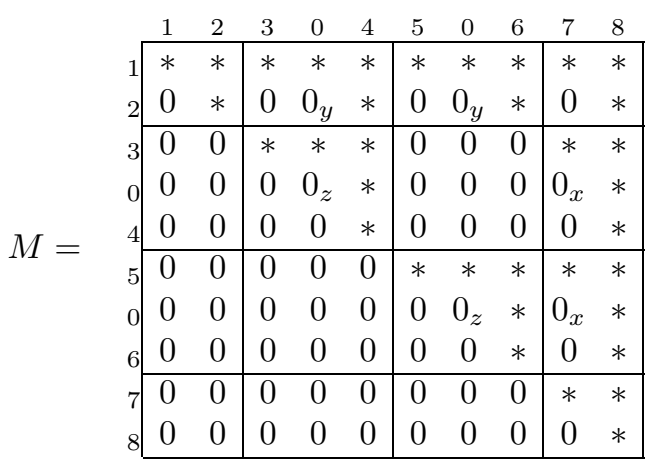

The blocks denoted by $0_{x}$ (respectively $0_{y}$ or $0_{z}$ ) are the so-called adjoint blocks, which means there is a unique block $x$ (respectively $y$ or $z$ ) operating on both of them, and thus only one block from an adjoint pair can be reduced to zero.

Nontrivial endomorphisms. Let us analyze matrices $\frac{d F_{k}}{d z_{0}}(0), f_{x}(0)$ and $f_{y}(0)$ looking for an endomorphism. Taking into account equations $F_{k}(0) \mu_{k}(0)=$ $\mu_{k}(0) f(0)$ for $k=2,3$ we see that there are nonzero matrices $f_{x}(0)$ and $f_{y}(0)$ leaving the matrices $\mu_{\varepsilon_{2}}(0)$ and $\mu_{\varepsilon_{3}}(0)$ in the zero form. Hence, as in the case of a tacnode curve, there are places $(i, j)$, where zero can be obtained in two or more different ways (that is, if $s_{i} \cdot s_{j}>0$, then there exists a nonscalar endomorphism). 
TABLE 4

\begin{tabular}{|c|c|c|c|c|}
\hline & condition & set $I$ & dimension vector $\$$ & state \\
\hline \hline 1. & $r \geq \bar{d}$ & $(1,2,3,5)$ & $\left(r-\bar{d}, \bar{d}_{3}, \bar{d}_{2}, \bar{d}_{1}\right)$ & $A^{+}$ \\
\hline $1^{\prime}$. & $\bar{d}>2 r$ & $(4,6,7,8)$ & $\left(r-\bar{d}_{1}, r-\bar{d}_{2}, r-\bar{d}_{3}, \bar{d}-2 r\right)$ & $A^{-}$ \\
\hline \hline 2. & $\begin{array}{c}\bar{d}>r>\bar{d}_{i}+\bar{d}_{j} \\
\text { for all } i, j \in\{1,2,3\} ;\end{array}$ & $(2,3,5,0)$ & $\left(r-\left(\bar{d}_{1}+\bar{d}_{2}\right), r-\left(\bar{d}_{1}+\bar{d}_{3}\right), r-\left(\bar{d}_{2}+\bar{d}_{3}\right), \bar{d}-r\right)$ & $A^{-}$ \\
\hline $2^{\prime}$. & $\begin{array}{c}\bar{d}_{i}+\bar{d}_{j}>r \text { and } 2 r>\bar{d} \\
\text { for all } i, j \in\{1,2,3\} ;\end{array}$ & $(0,4,6,7)$ & $\left(2 r-\bar{d},\left(\bar{d}_{2}+\bar{d}_{3}\right)-r,\left(\bar{d}_{1}+\bar{d}_{3}\right)-r,\left(\bar{d}_{1}+\bar{d}_{2}\right)-r\right)$ & $A^{+}$ \\
\hline \hline 3. & $\begin{array}{c}\left(\bar{d}_{2}+\bar{d}_{3}\right)>r \text { and } \\
r>\left(\bar{d}_{1}+\bar{d}_{2}\right),\left(\bar{d}_{1}+\bar{d}_{3}\right)\end{array}$ & $(2,3,0,4)$ & $\left(r-\left(\bar{d}_{1}+\bar{d}_{2}\right), r-\left(\bar{d}_{1}+\bar{d}_{3}\right), \bar{d}_{1},\left(\bar{d}_{2}+\bar{d}_{3}\right)-r\right)$ & $B^{-}(0)$ \\
\hline $3^{\prime}$. & $\begin{array}{c}\left(\bar{d}_{1}+\bar{d}_{2}\right),\left(\bar{d}_{1}+\bar{d}_{3}\right)>r \\
r>\left(\bar{d}_{2}+\bar{d}_{3}\right)\end{array}$ & $(5,0,6,7)$ & $\left(r-\left(\bar{d}_{2}+\bar{d}_{3}\right), r-\bar{d}_{1},\left(\bar{d}_{1}+\bar{d}_{3}\right)-r,\left(\bar{d}_{1}+\bar{d}_{2}\right)-r\right)$ & $B^{+}(0)$ \\
\hline \hline 4. & $\begin{array}{c}\left(\bar{d}_{1}+\bar{d}_{3}\right),\left(\bar{d}_{2}+\bar{d}_{3}\right)>r \\
\text { and } r>\left(\bar{d}_{1}+\bar{d}_{2}\right),\end{array}$ & $(2,0,4,6)$ & $\left(r-\left(\bar{d}_{1}+\bar{d}_{2}\right), r-\bar{d}_{3},\left(\bar{d}_{2}+\bar{d}_{3}\right)-r,\left(\bar{d}_{1}+\bar{d}_{3}\right)-r\right)$ & $B^{+}(0)$ \\
\hline $4^{\prime}$ & $\begin{array}{c}\left(\bar{d}_{1}+\bar{d}_{2}\right)>r \text { and } \\
r>\left(\bar{d}_{1}+\bar{d}_{3}\right),\left(\bar{d}_{2}+\bar{d}_{3}\right)\end{array}$ & $(3,5,0,7)$ & $\left(r-\left(\bar{d}_{1}+\bar{d}_{3}\right), r-\left(\bar{d}_{2}+\bar{d}_{3}\right), \bar{d}_{3},\left(\bar{d}_{1}+\bar{d}_{2}\right)-r\right)$ & $B^{-}(0)$ \\
\hline \hline 5. & $\begin{array}{c}\left(\bar{d}_{1}+\bar{d}_{3}\right)>r \text { and } \\
r>\left(\bar{d}_{1}+\bar{d}_{2}\right),\left(\bar{d}_{2}+\bar{d}_{3}\right)\end{array}$ & $(2,5,0,6)$ & $\left(r-\left(\bar{d}_{1}+\bar{d}_{2}\right), r-\left(\bar{d}_{2}+\bar{d}_{3}\right), \bar{d}_{2},\left(\bar{d}_{1}+\bar{d}_{3}\right)-r\right)$ & $B^{-}(0)$ \\
\hline $5^{\prime}$. & $\begin{array}{c}\left(\bar{d}_{1}+\bar{d}_{2}\right),\left(\bar{d}_{2}+\bar{d}_{3}\right)>r \\
\text { and } r>\left(\bar{d}_{1}+\bar{d}_{3}\right)\end{array}$ & $(2,0,4,7)$ & $\left(r-\left(\bar{d}_{1}+\bar{d}_{3}\right), r-\bar{d}_{2},\left(\bar{d}_{2}+\bar{d}_{3}\right)-r,\left(\bar{d}_{1}+\bar{d}_{2}\right)-r\right)$ & $B^{+}(0)$ \\
\hline
\end{tabular}

The diagram of mutually excluding blocks is almost the same as the diagram (6.7):

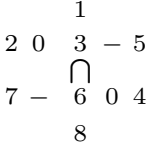

where intersections

- $1,2 \cap 7,8$ are induced by $\frac{d F_{1}}{d z_{0}}(0)$ and $\frac{d F_{2}}{d z_{0}}(0)$;

- $1,3 \cap 6,8$ by $\frac{d F_{1}}{d z_{0}}(0)$ and $\frac{d F_{3}}{d z_{0}}(0)$;

- $1,5 \cap 4,8$ by $\frac{d F_{2}}{d z_{0}}(0)$ and $\frac{d F_{3}}{d z_{0}}(0)$;

- and entries $(1,0)$ and $(0,8)$ link all three matrices $\frac{d F_{1}}{d z_{0}}(0), \frac{d F_{2}}{d z_{0}}(0)$ and $\frac{d F_{3}}{d z_{0}}(0)$. In Table 4 we present the maximal tuples $I=\left\{i_{1}, i_{2}, i_{3}, i_{4}\right\}$, interpret the dimension vector $\$$ in terms of rank and multidegree and specialize matrices that we get in each case.

By $A^{\sigma}$ and $B^{\sigma}(j)$ we denote the matrix problems given by the following coincidence matrices $M$ :

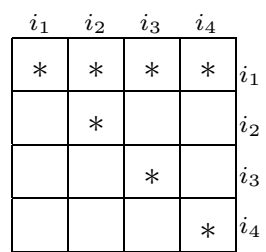

$A^{+}$

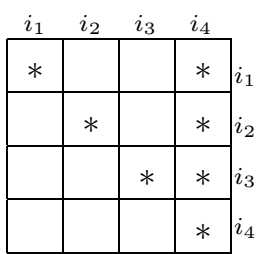

A-

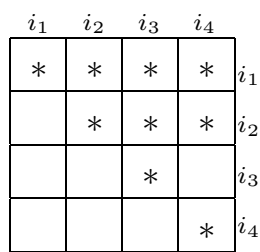

$B^{+}\left(i_{2}\right)$

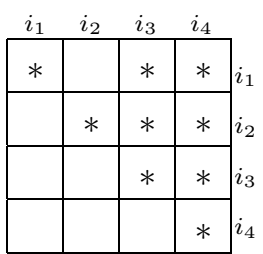

$B^{-}\left(i_{3}\right)$. 
As usual, the matrix problems are $M \mapsto S M S^{-1}$ modulo empty spaces and the transformation $S$ has the form transposed to $M$.

\section{Matrix PROBlems}

In this section we use the technique of boxes and follow the notation of BD09. From now on let $\mathfrak{A}$ be a Roiter box and $(Q, \partial)$ its differential biquiver, where $Q=\left(I, Q_{0}, Q_{1}\right)$ with the set of vertices $I$ and the sets of solid and dotted arrows, respectively $Q_{0}$ and $Q_{1}$. Let $\mathfrak{A}$-mod be the category of finite-dimensional $\mathfrak{A}$-modules and $\mathrm{Br}_{\mathfrak{A}}$ its full subcategory of bricks. For details concerning boxes we also refer to Dro01 and Bod07. Summarizing previous sections we conclude that our approach provides a full and dense functor $\mathrm{VB}_{E} \stackrel{\sim}{\longrightarrow} \operatorname{Tr}_{E} \longrightarrow \mathrm{MP}_{E}$ and the primary reduction is an equivalence of categories $\mathrm{MP}_{E}^{s}(\mathbb{r}) \stackrel{\sim}{\longrightarrow} \operatorname{Br}_{\mathfrak{A}}(\mathbb{s})$, for some special box $\mathfrak{A}$ and dimension vector $\mathbb{s}$. The composition of these functors yields an equivalence $\operatorname{VB}_{E}^{s}(r, \mathbb{d}) \stackrel{\sim}{\longrightarrow} \operatorname{Br}_{\mathfrak{A}}(\$)$, where both the box $\mathfrak{A}$ and the tuple $s$ are uniquely defined by the curve $E$, the rank $r$ and the multidegree $d$.

In most situations it is useful to present a representation $M$ as a block matrix with the block $M(x)$ in the place $(i, j)$ for $x \in Q_{0}(j, i)$. As in the previous sections, with a little abuse of notation, we write the matrices $M$ and $S$ in a form of a table with $x$ on the $(i, j)$-entry instead of $M(x)$. In accordance with Section 6 we denote an identity-block and a zero-block by " $\mathbb{1}$ " and " 0 " respectively. Thus we adjust our former notation to that of the theory of boxes.

Class of BC-boxes. A box $\mathfrak{A}$ with the differential biquiver $(Q, \partial)$ is of $\mathrm{BC}$-type if its solid arrows form an $I \times I$ matrix. There are two total orders on the set $I$ : a row order denoted by $<_{r}$ and a column order denoted by $<_{c}$. The set of dotted arrows $Q_{1}$ consists of two subsets: $\left\{u \in Q_{1}(k, j) \mid j>{ }_{r} k\right\}$ and $\left\{v \in Q_{1}(i, l) \mid l>{ }_{c} i\right\}$. For each $x \in Q_{0}(i, j)$, the differential is

$$
\partial(x)=\sum_{l<{ }_{c} i} x^{\prime} v-\sum_{j<{ }_{r} k} u x^{\prime \prime},
$$

where $x^{\prime} \in Q_{0}(l, j)$ and $x^{\prime \prime} \in Q_{0}(i, k)$ are uniquely defined as the entries $(j, l)$ and $(k, i)$ of the matrix $I \times I$. Such boxes can be presented via matrices $M$ and $\left(S, S^{\prime}\right)$ and matrix multiplications: $M \mapsto S M\left(S^{\prime}\right)^{-1}$, where

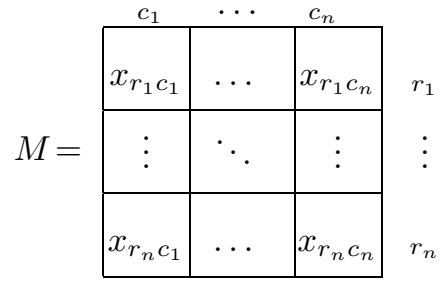

$\left(S, S^{\prime}\right)=\left(\begin{array}{|c|c|c||c|c|c|}\hline w_{r_{1}} & 0 & 0 & w_{c_{1}} & 0 & 0 \\ \hline \vdots & \ddots & 0 & \vdots & \ddots & 0 \\ \hline u_{r_{n} r_{1}} & \ldots & w_{r_{n}} & v_{c_{n} c_{1}} & \ldots & w_{c_{n}} \\ \hline\end{array}\right.$

and $\left(r_{1}, \ldots, r_{n}\right)$ and $\left(c_{1}, \ldots, c_{n}\right)$ are orders $<_{r}$ and $<_{c}$ on $I$, i.e. $r_{1}<_{r} r_{2}<_{r} \cdots<_{r}$ $r_{n}$ and $c_{1}<_{c} c_{2}<_{c} \cdots<_{c} c_{n}$. The reduced matrix problem for a nodal curve from Subsection 6.1 as well as all the problems $A^{+}, A^{-}$and $C$ from Subsections 6.3 and 6.5 are of BC-type. Note that BC-matrix problems are examples of bunches of chains. 
Class of BT-boxes. A box $\mathfrak{A}$ with the differential biquiver $(Q, \partial)$ is of BT-type if there exists a set of distinguished loops: $\mathfrak{a}:=\left\{a_{i} \in Q_{0}(i, i) \mid i \in I\right\}$, an injective map: $v: Q_{0} \backslash \mathfrak{a} \hookrightarrow Q_{1}$, mapping a solid arrow $a: i \rightarrow j$ to an opposite directed dotted arrow $v_{a}:=v(a): j \cdots>i$, and for each distinguished loop $a_{i} \in \mathfrak{a}$ we have

$$
\partial a_{i}=\sum_{c: \cdot \rightarrow i} c \cdot v_{c}-\sum_{d: i \rightarrow \cdot} v_{d} \cdot d .
$$

The class of BT-boxes was studied in detail in BD09. The main property is that a connected BT-box with more than one vertex is wild but brick-tame. However, here we do not use any theoretical results. Our arguments are based on the concrete calculations for BT-boxes with at most four vertices. For a box $\mathfrak{A}$ of BT-type its biquiver $Q$ can be encoded as follows: a vertex $i \in I$ is denoted by a bullet •; on the set of vertices we draw the graph with arrows $Q_{0} \backslash \mathfrak{a}$. Such a system of notation becomes quite useful since in most of our cases it is clear how to recover the differential.

The BT-box $\mathfrak{A}$ obtained in Subsection 6.2 for a cuspidal cubic curve is ${ }^{1} \bullet \longleftarrow \bullet^{2}$. The problems on three vertices $A^{+}$and $A^{-}$from Subsection 6.4 and the problems on four vertices: $A^{+}, A^{-}, B^{+}(j)$ and $B^{-}(i)$ from Subsection 6.6 are also of BT-type:

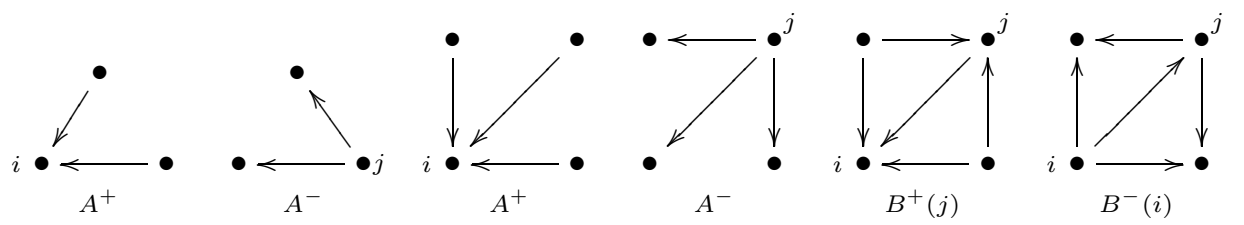

Remark 7.1. The listed BT-boxes and those that appear in the following sections determine partially ordered sets $(I, \prec)$, by the rule $i \prec j$ if there exists $x \in Q_{0}(j, i)$. In most of our cases a poset defines a box; however, in general, it does not provide enough information to recover the differential. On the other hand, a pair of linear orders $<_{r}$ and $<_{c}$ in the definition of a BC-box determine a partial order $\prec$ by the rule $i \prec j$ if $i<_{r} j$ and $i<_{c} j$. Posets obtained in such a way relay BC and BT-boxes. Moreover, for the BT-box they determine the canonical minimal edge $(i j)$, where $i$ is the minimal with respect to the total order $<_{r}$ and $j$ is the maximal with respect to $<_{c}$. Thus having a fixed dimension vector $\$$, not only for a BC-box but also for the corresponding BT-box, we have the canonical course of reduction.

Bricks and small reduction. Boxes of BC and BT-types possess a common property. The following proposition allows us to replace the usual matrix reduction by the small one.

Proposition 7.2. Let $\mathfrak{A}$ be a box of $\mathrm{BC}$ or $\mathrm{BT}$ type, $b: i \rightarrow j$ its minimal edge and $M$ a brick. Then $M(b)$ has maximal rank.

Proof. Let $\mathfrak{A}$ be a box of BC-type. Since $\mathfrak{A}$ is an example of bunches of chains, we can assume that $M$ is reduced to its canonical form. Also assume that $M(b)=$ $\left(\begin{array}{ll}0 & 0 \\ I & 0\end{array}\right)$. Let the rows and columns of $M$ be ordered $1, \ldots, R$. For a place $t \in$ $\{1, \ldots, R\}$, by $r(t)$ and $c(t)$ we denote the row-block and the column-block containing $t$. For example, since rows and columns are ordered, we have $r(1)=j$ and $c(R)=i$. If $M$ is invertible, then there exist places $m$ and $n$ such that $M_{1 m}=M_{n R}=1$ and all the other entries in the first row and the last ( $R$-th) column are zero. A nonscalar endomorphism $\left(S, S^{\prime}\right)$ of $M$ can be constructed by taking nonzero $S_{n 1}=-S_{R m}^{\prime}$, diagonal entries to be, for example, 1 and all the other 
nondiagonal entries to be zero. Since $c(m)<_{c} i$ and $r(n)>_{r} j$ the block $S_{r(n) r(1)}$ containing the entry $S_{n 1}$ and the block $S_{c(R) c(m)}^{\prime}$ containing the entry $S_{R m}^{\prime}$ are nonempty.

If $\mathfrak{A}$ is a box of BT-type, then after a step of minimal edge reduction there is a dotted arrow which is not involved in any differential and hence there is a nonscalar endomorphism (for details, see [BD09, Lemma 3.1]).

7.1. Small reduction automaton. Recall that an automaton is an oriented graph on the set of vertices called states, whose arrows are transitions from a state to a state. In our case the states are the matrix problems and the transitions encode canonical steps of the reduction.

Definition 7.3. A small-reduction automaton is an oriented graph $\Gamma$, where

- the set of states $\Gamma_{0}$ is finite and consists of boxes $\mathfrak{A}$, whose differential biquivers have the same finite set of vertices $I$.

- The set of transitions $\Gamma_{1}$ is a subset of $I \times I$.

- For a minimal solid arrow either $j \rightarrow i$ or $i \rightarrow j$ the transition $(i j): \mathfrak{A} \rightarrow \mathfrak{A}^{\prime}$ acts on the space of sizes $\mathbb{N}^{|I|}$ as : $\mathbb{\$} \mapsto \mathbb{s}^{\prime}$, where $s_{k}^{\prime}=s_{k}$ for $k \neq i$ and $s_{i} \mapsto s_{i}-s_{j}$, provided $s_{i}>s_{j}$.

A sequence $p:=\left(i_{n} j_{n}\right) \ldots\left(i_{2} j_{2}\right)\left(i_{1} j_{1}\right)$ of transitions is called a path if the target of $\left(i_{k} j_{k}\right)$ coincides with the source of $\left(i_{k+1} j_{k+1}\right)$. A path operates on the set of sizes: $p: \mathbb{s} \mapsto \mathbb{s}^{\prime}$, where $\mathbb{s} \geq \mathbb{s}^{\prime}$; i.e. $s_{i} \geq s_{i}^{\prime}$ for all $i \in I$. Two paths $p_{1}$ and $p_{2}$ with a common source and a common target are called equivalent if for any tuple of sizes $\$ \in \mathbb{N}^{I}$ we have $p_{1}(\$)=p_{2}(\$)$. The semigroup of paths modulo the equivalence relation is called the semigroup of the automaton.

Principal states. Let $\Gamma$ be an automaton of small reduction starting from one of the boxes from Tables 24. A state $\mathfrak{A} \in \Gamma_{0}$ is called principal if it can be interpreted in terms of vector bundles $\operatorname{Br}_{\mathfrak{A}}(\mathbb{s}) \cong \mathrm{VB}_{E}^{s}(r, \mathbb{d})$. We show that for a rank $r$ and a multidegree $\mathbb{d}$ such that $\operatorname{gcd}(r, d)=1$ there exists a path $p$ on $\Gamma$ connecting principal states $\mathfrak{A}$ and $\mathfrak{A}^{\prime}$ such that

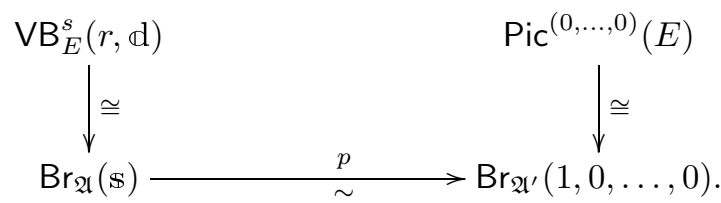

Then a canonical form of a simple vector bundle can be constructed as follows.

7.2. Algorithm. Let $E$ be a reduced plane degeneration of an elliptic curve with $N$ components, let $(r, \mathbb{d}) \in \mathbb{N} \times \mathbb{Z}^{N}$ be a tuple of integers, such that $\operatorname{gcd}(r, d)=1$, where $d=\sum_{k=1}^{N} d_{k}$, and let $\lambda \in \mathbb{k}$ be a continuous parameter.

(1) Use one of the Tables 2, 3 or 4 (with respect to the type of $E$ ) to recover the matrix problem $\mathrm{Br}_{\mathfrak{A}}$ and the dimension vector $\mathbb{s} \in \mathbb{N}^{N+1}$ from $(r, \mathbb{d})$.

(2) Take $\operatorname{Br}_{\mathfrak{A}}(\$)$ as the input data for the corresponding small-reduction automaton. Choose a path $p$ on it such that $p(s)=(1,0, \ldots, 0)$.

(3) Starting with the one-dimensional matrix $\lambda \in \operatorname{Br}_{\mathbb{k}[t]}(1)$, reverse the course of reduction along the path $p$. This way, step-by-step recover the canonical form $M(\lambda)=p^{-1}(\lambda) \in \mathrm{Br}_{\mathfrak{A}}(\mathbb{s}) \cong \operatorname{VB}_{E}^{s}(r, \mathbb{d})$. 


\section{Small Reduction for nOdal AND CUSPidal CUbiC CURVES}

The categories obtained in Subsections 6.1 and 6.2 can be interpreted as the categories $\mathfrak{A}$ - $\bmod \left(s_{1}, s_{2}\right)$, where $\mathfrak{A}$ are boxes of either $\mathrm{BC}$ and BT-types. In order to illustrate the language of boxes we present $\mathfrak{A}$ for a nodal curve as a differential biquiver, despite the agreement to present BC-boxes by tables:

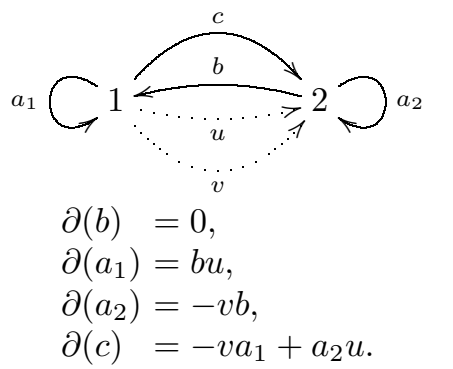

and

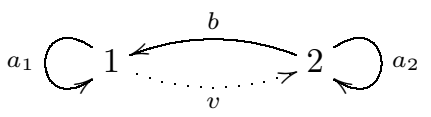

$$
\begin{aligned}
& \partial(b)=0 \\
& \partial\left(a_{1}\right)=b v \\
& \partial\left(a_{2}\right)=-v b .
\end{aligned}
$$

In both cases the steps of small reduction are $\mathfrak{A} \stackrel{(12),(21)}{\longrightarrow} \mathfrak{A}$. In other words, both problems are self-reproducing, and the small-reduction automaton is

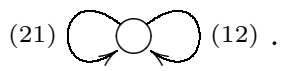

The transitions act on sizes as $(12):\left(s_{1}, s_{2}\right) \mapsto\left(s_{1}-s_{2}, s_{2}\right)$ if $s_{1} \geq s_{2}$ and (21): $\left(s_{1}, s_{2}\right) \mapsto\left(s_{1}, s_{2}-s_{1}\right)$ if $s_{1}<s_{2}$. In terms or rank and degree we get

$$
(12): \mathrm{VB}_{E}^{s}(r, \bar{d}) \rightarrow \mathrm{VB}_{E}^{s}(r-\bar{d}, \bar{d}) \text { and }(21): \mathrm{VB}_{E}^{s}(r, \bar{d}) \rightarrow \mathrm{VB}_{E}^{s}(\bar{d}, 2 \bar{d}-r)
$$

That implies the statement of Theorem 1.2 for irreducible cubic curves.

Remark 8.1. The semigroup of paths $\langle(21),(12)\rangle$, generates an $\operatorname{SL}(2, \mathbb{Z})$-action on the set of discrete parameters $\left(s_{1}, s_{2}\right)$.

Remark 8.2. The group generated by Seidel-Thomas spherical twists $\left\langle\mathrm{T}_{\mathcal{O}}, \mathrm{T}_{\mathbb{k}\left(p_{0}\right)}\right\rangle$ acts as $\mathrm{SL}(2, \mathbb{Z})$ on the $K$-group, or what is equivalent, on the rank and degree $(r, d) \in \operatorname{Hom}_{\mathbb{Z}}\left(K_{0}(E), \mathbb{Z}\right)$. For singular Weierstraß curves the action of the reduction automaton on discrete parameters $(r, \bar{d})$ can be interpreted in terms of FourierMukai transforms; namely, (12) acts as $\mathrm{T}_{\mathcal{O}}$ and (21) acts as $\mathrm{T}_{\mathbb{k}\left(p_{0}\right)}^{2} \mathbb{F}$, where

$$
\mathbb{F}=\mathrm{T}_{\mathbb{k}\left(p_{0}\right)} \mathrm{T}_{\mathcal{O}} \mathrm{T}_{\mathbb{k}\left(p_{0}\right)}[-1] \text {. }
$$

For a singular Weierstraß curve $E$ in [BD09, Corollary 5.11], Burban and Drozd constructed a fully-faithful functor $\operatorname{Perf}(E) \hookrightarrow D^{b}(B)$, where $B$ is the so-called ButlerBurt algebra associated to the matrix problem $\mathrm{MP}_{E}$ as introduced in [BB91]. The combinatorics of bricks over $B$ is the same as that over $\mathfrak{A}$. There is strong evidence that the small reductions (12) and (21) are shadows of some derived autoequivalences of the derived category $D^{b}(B)$.

Remark 8.3. Let us stress that for reductions (8.2) we take $\operatorname{VB}_{E}^{s}(r, \bar{d})$ with $0 \leq$ $\bar{d}<r$, or in other words, the full subcategory of vector bundles $\mathcal{E} \in \mathrm{VB}_{E}^{s}$ with the normalization $\widetilde{\mathcal{E}}=\mathcal{O}_{\mathbb{P}^{1}}^{r-\bar{d}} \oplus \mathcal{O}_{\mathbb{P}^{1}}^{\bar{d}}(1)$. If the degree $d$ is arbitrary, we identify $\operatorname{VB}_{E}^{s}(r, d)$ with $\operatorname{VB}_{E}^{s}(r, \bar{d})$, where $\bar{d}=d \bmod r$, using the Picard group action. For instance, to proceed with the reduction after the (12)-step, we have to replace the category $\operatorname{VB}_{E}^{s}(r-\bar{d}, \bar{d})$ by $\operatorname{VB}_{E}^{s}(r-\bar{d}, \bar{d} \bmod (r-\bar{d}))$. 


\section{Small Reduction for Kodaira fibers $\mathrm{I}_{2}$ AND III}

In Subsections 6.3 and 6.4 we obtained an equivalence $\operatorname{MP}^{s}(r, \mathbb{d}) \stackrel{\cong}{\longrightarrow} \operatorname{Br}_{\mathfrak{A}}(\mathbb{s})$, where the box $\mathfrak{A}$ is the configuration $A^{\sigma}$, of BC or BT-type, $\sigma \in\{+,-\}$ depending on whether $r>\bar{d}$ or $r<\bar{d}$. Applying a small reduction to the box $A^{\sigma}$ we obtain another configuration on 3 blocks, defined by the standard numeration of blocks $(1,2,3)$. We denote this type of box by $B$. In the BC-case we get:

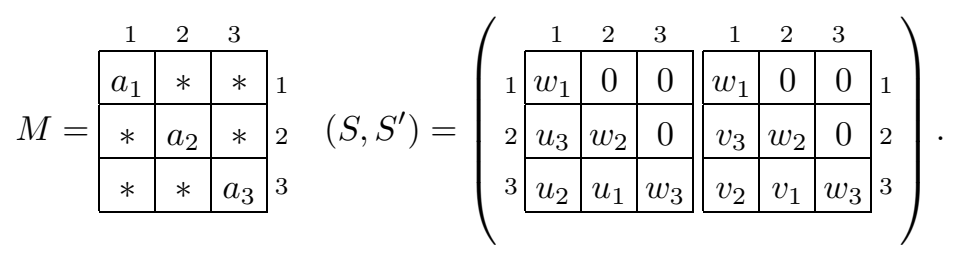

As was mentioned in Remark 7.1 column and row-orders define a poset. Configurations $A^{+}, A^{-}$and $B$ determine respectively the posets

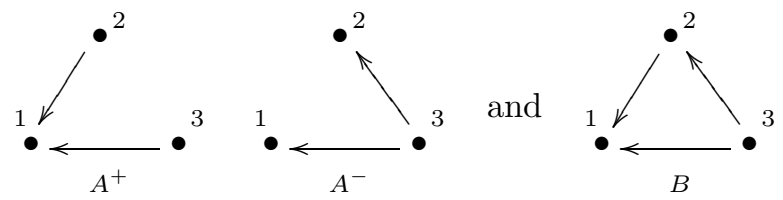

Let's illustrate by an example how to associate a BT-differential biquiver to a poset. For $A^{-}$and $B$ we have respectively:

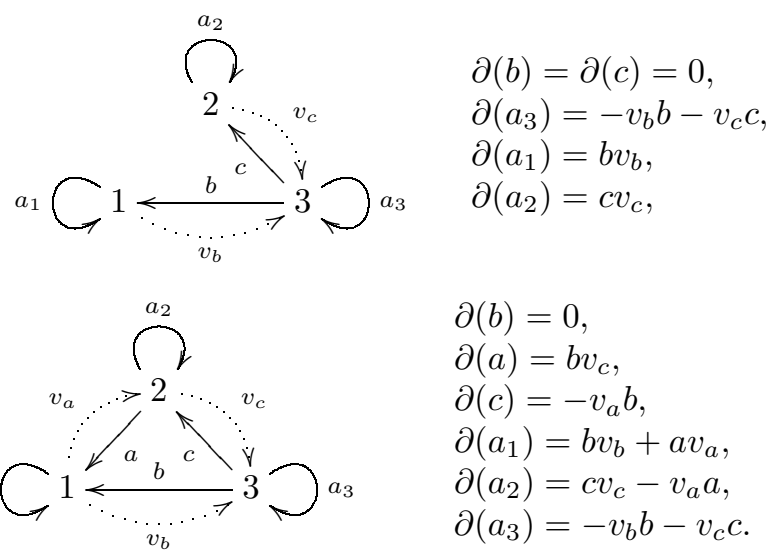

In Subsection 6.4 we obtained an equivalence $\operatorname{MP}^{s}\left(r, d_{1}, d_{2}\right) \stackrel{\cong}{\longrightarrow} \operatorname{Br}_{\mathfrak{A}}\left(s_{1}, s_{2}, s_{3}\right)$, where $\mathfrak{A}$ was a BT-box of type either $A^{+}$or $A^{-}$. The small reduction automaton starting at, let us say $A^{+}$, is

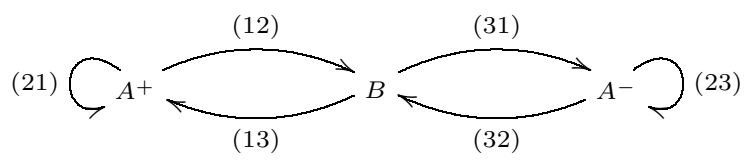

This is the small reduction automaton for a cycle of two lines, which is also the canonical one for a tacnode curve. We claim that the reduction can terminate only 
at the states $A^{+}$and $A^{-}$, which are principal. Indeed, assume that we have the box $B$ with sizes $s_{1}=s_{3}$. Then the matrix can be reduced to the canonical form:

\begin{tabular}{|l|c|c|c}
1 & 2 & \multicolumn{2}{|c}{3} \\
\hline 0 & 0 & $\mathbb{1}$ & \multirow{1}{*}{1} \\
\cline { 1 - 3 } 0 & $J_{1}$ & 0 & \multirow{2}{2}{2} \\
\cline { 1 - 3 }$J_{2}$ & 0 & 0 & 3 \\
\end{tabular}

where $J_{1}$ and $J_{2}$ are Jordan cells with nonzero eigenvalues. It is quite obvious that this matrix is decomposable. Analogously, in the case of Kodaira fiber III, the reduction can terminate only at a state of type $A$. Indeed, if $s_{1}=s_{3}$, then the configuration $B$ produces a splitting, and for $A^{+}$we get the problem $\operatorname{Br}_{\mathfrak{A}}\left(s_{1}, s_{2}\right)$, where $\mathfrak{A}$ is the box as for a cuspidal cubic curve with sizes $\left(s_{1}, s_{2}, s_{3}\right) \mapsto\left(s_{1}, s_{2}\right)$ :

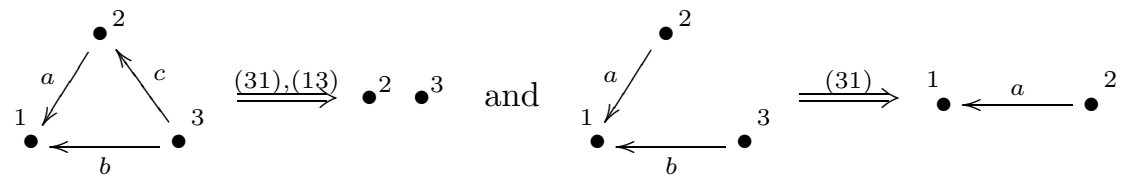

By gluing paths we can construct the automaton on principal states:

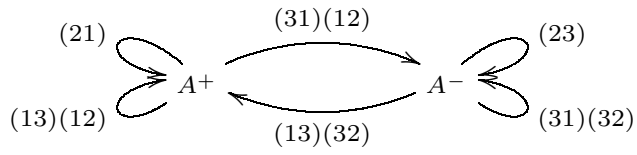

For a principal configuration $A^{\sigma}$ we introduce its new discrete parameters $(\alpha, \beta)$. For $A^{+}$let $(\alpha, \beta):=\left(s_{1}, s_{2}+s_{3}\right)$ and $(\alpha, \beta):=\left(s_{1}+s_{2}, s_{3}\right)$ for $A^{-}$.

Lemma 9.1. Let $p: A^{\sigma} \rightarrow A^{\sigma^{\prime}}$ be a path on the principal automaton (9.4) taking $\mathbb{s} \mapsto \mathbb{s}^{\prime}$ and respectively $(\alpha, \beta) \rightarrow\left(\alpha^{\prime}, \beta^{\prime}\right)$. Then $\operatorname{gcd}(\alpha, \beta)=\operatorname{gcd}\left(\alpha^{\prime}, \beta^{\prime}\right)$.

Proof. It is sufficient to prove the statement on the following transitions: (23), $(32)(31): A^{-} \rightarrow A^{-}$and $(13)(32): A^{-} \rightarrow A^{+}$. Indeed, we have

$$
\begin{aligned}
& (23):\left(s_{1}, s_{2}, s_{3}\right) \mapsto\left(s_{1}, s_{2}-s_{3}, s_{3}\right) \text { and hence }(\alpha, \beta) \mapsto(\alpha-\beta, \beta) ; \\
& (32)(31):\left(s_{1}, s_{2}, s_{3}\right) \mapsto\left(s_{1}, s_{2}, s_{3}-\left(s_{1}+s_{2}\right)\right) \text { and }(\alpha, \beta) \mapsto(\alpha, \beta-\alpha) ; \\
& (13)(32):\left(s_{1}, s_{2}, s_{3}\right) \mapsto\left(s_{1}+s_{2}-s_{3}, s_{2}, s_{3}-s_{2}\right) \text { and }(\alpha, \beta) \mapsto(\alpha-\beta, \beta) .
\end{aligned}
$$

Let $\operatorname{VB}_{E}^{s}(r, \mathbb{d}) \cong \mathrm{VB}_{E}^{s}\left(r^{\prime}, \mathbb{d}^{\prime}\right)$ be a functorial bijection obtained by the course of small reductions along the path $p$. Replacing the dimension vector $\$$ by the tuple $(r, \mathbb{d})$ using Table 2 we obtain $(\alpha, \beta)=(r-d \bmod r, d \bmod r)$. If $\operatorname{gcd}(r, d)=1$, at the end of the reduction we get $\operatorname{VB}_{E}^{s}(r, \mathbb{d}) \stackrel{\cong}{\longrightarrow} \mathrm{Pic}^{(0,0)}(E)$, and there are no bricks otherwise. Hence, Lemma 9.1 implies Theorem 1.2 for curves $\mathrm{I}_{2}$ and III.

\section{Small Reduction FOR Kodaira Fibers $\mathrm{I}_{3}$ AND IV}

In Subsection 6.5 we obtained some equivalences $\mathrm{MP}^{s}(r, \mathbb{d}) \stackrel{\cong}{\longrightarrow} \operatorname{Br}_{\mathfrak{A}}(\mathbb{\$})$, where $\mathbb{s} \in \mathbb{N}^{4}$ and $\mathfrak{A}$ is a BC-box of type $A^{+}, A^{-}$or $C$. To fix the notation we rewrite 
the configurations for the set of vertices $I=\{1,2,3,4\}$. Then a small reduction automaton starting from the configuration $A^{+}$is as follows:

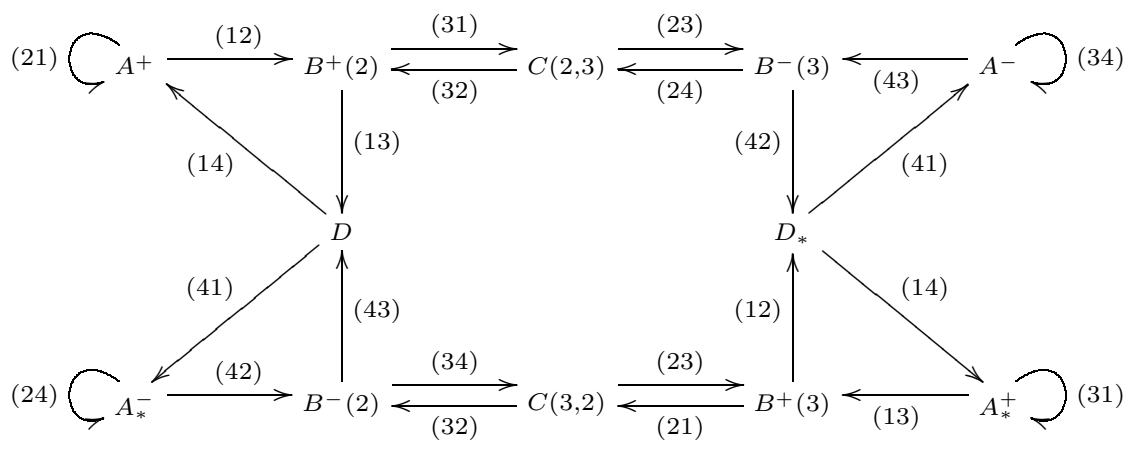

Let us explain the notation: the configurations of type $A$ are
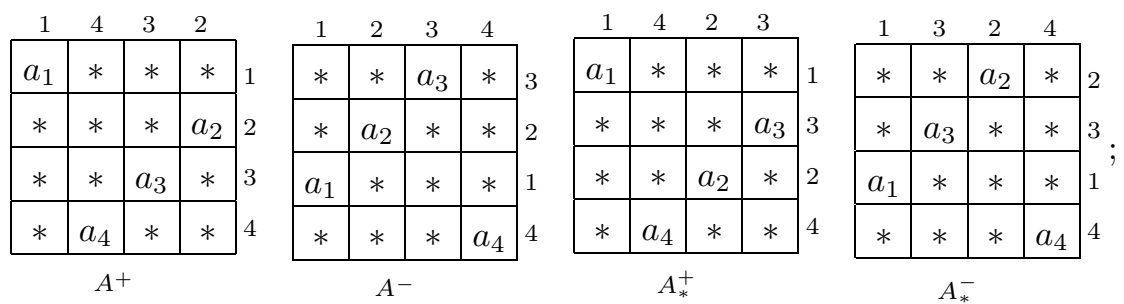

the configurations of type $B$ are
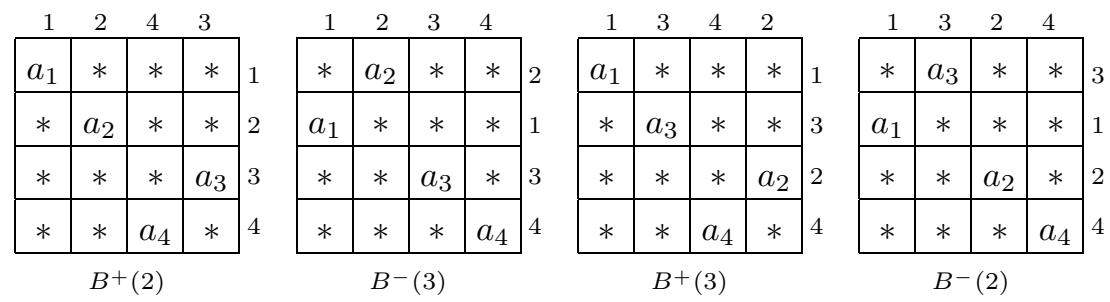

and the configurations of types $C$ and $D$ are

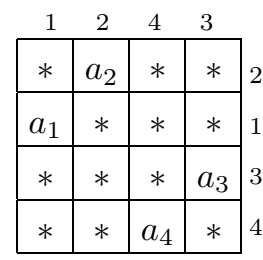

$C(2,3)$

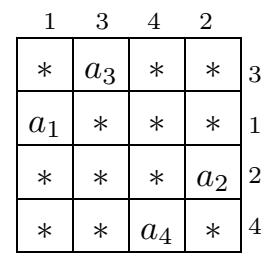

$C(3,2)$
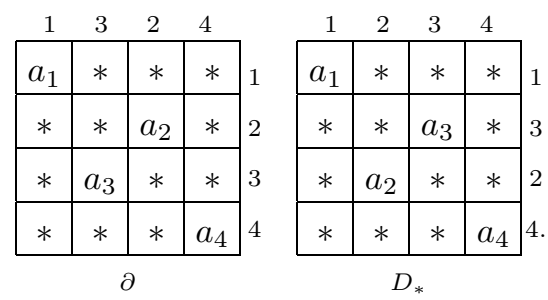

In Subsection 6.6 we obtained equivalences $\operatorname{MP}^{s}(r, \mathbb{d}) \stackrel{\cong}{\longrightarrow} \operatorname{Br}_{\mathfrak{A}}(\mathbb{s}), \mathbb{s} \in \mathbb{N}^{4}$, where $\mathfrak{A}$ is a BC-box of type $A^{+}, A^{-}$or $B$. As explained in Remark 7.1, the boxes of BC and BT types are related. Therefore the canonical small reduction automaton for Kodaira fiber IV can be obtained from the automaton 10.1) by gluing states $A^{\sigma}$ 
with $A_{*}^{\sigma}$ and $D$ with $D_{*}$ :

$(10.2)$

(31)

(21)

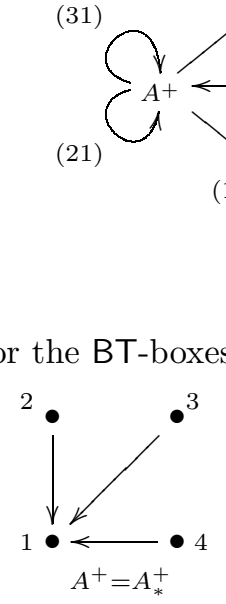

(12)

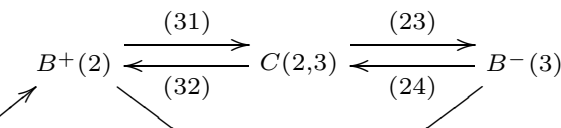

(13)
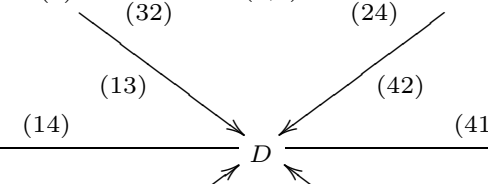

(14)
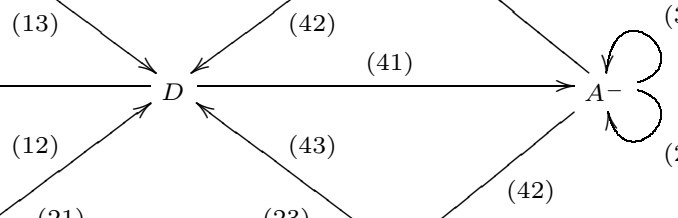

(1)

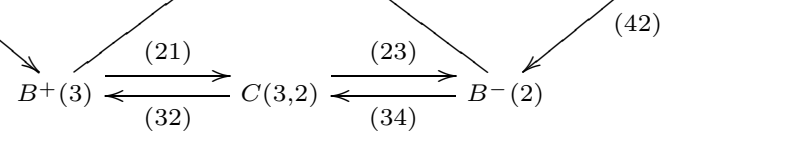

For the BT-boxes we have
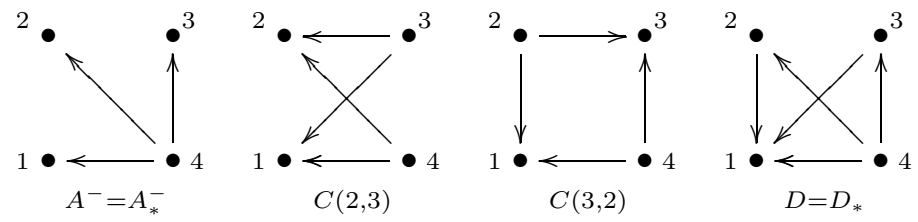

and four configurations of type $B$ :
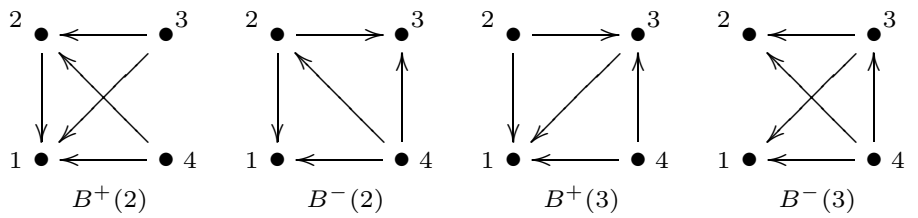

All edge arrows of the poset $A$ are minimal. The posets $B$ and $D$ are of height 2. Their differential biquivers are uniquely defined by the rule as follows: for any triangle $i \prec j \prec k$ with arrows $a: j \rightarrow i, b: k \rightarrow i$, and $c: k \rightarrow j$, the arrow $b$ is minimal, $\partial(a)=\sum_{k} b v_{c}+\phi$ and $\partial(c)=-\sum_{i} v_{a} b+\psi$, where $\phi$ and $\psi$ are summands coming from the other triangles. That is, in a triangle the differential is as in (9.1) of type $B$. For the poset $C$ one should additionally give a pair of minimal edges: for $C(2,3)$ they are $3 \rightarrow 2$ and $4 \rightarrow 1$, for $C(3,2)$ they are $2 \rightarrow 3$ and $4 \rightarrow 1$. The differentials of the other arrows consist of the paths of length 3 and degree one.

Rank and degree. For configurations of types $A, C$ and $B$, let $I_{\min } \subset I$ and $I_{\max } \subset I$ be the subsets of minimal and maximal vertices with respect to the partial order $\prec$. For a dimension vector $\mathbb{s} \in \mathbb{N}^{4}$ let us introduce new discrete parameters $(\alpha, \beta)$ :

- for a box of type either $A$ or $C$, define $\alpha:=\sum_{i \in I_{\min }} s_{i}$ and $\beta:=\sum_{k \in I_{\max }} s_{k}$;

- for a box $B^{\sigma}(j)$, define $\alpha:=s_{j}+\sum_{i \in I_{\min }} s_{i}$ and $\beta:=s_{j}+\sum_{k \in I_{\max }} s_{k}$.

Lemma 10.1. Let $\Gamma$ be the automaton either (10.1) or (10.2) and $p: \mathfrak{A} \rightarrow \mathfrak{A}^{\prime}$ a path on it connecting principal states $\mathfrak{A}$ and $\mathfrak{A}^{\prime}$ and taking $\mathbb{s} \mapsto \mathbb{s}^{\prime}$ and $(\alpha, \beta) \mapsto\left(\alpha^{\prime}, \beta^{\prime}\right)$. Then $\operatorname{gcd}(\alpha, \beta)=\operatorname{gcd}\left(\alpha^{\prime}, \beta^{\prime}\right)$. 
Proof. It is sufficient to check the statement on the shortest paths. For the transitions $A \rightarrow B$ or $C \rightarrow B$ we have $\left(\alpha^{\prime}, \beta^{\prime}\right)=(\alpha, \beta)$. For the transitions $A \rightarrow A$, $B \rightarrow C$ or a path of length two $B \rightarrow A$, we have

$$
\left(\alpha^{\prime}, \beta^{\prime}\right)= \begin{cases}(\alpha-\beta, \beta), & \text { if } \alpha \geq \beta \\ (\alpha, \beta-\alpha), & \text { if otherwise }\end{cases}
$$

That completes the proof.

To obtain the statement of Theorem 1.2 for the Kodaira fibers $\mathrm{I}_{3}$ and IV we should replace the pair $(\alpha, \beta)$ by the rank and degree $(r, \bar{d})$ using Tables 3 and 4 , In each case but cases 2 and $2^{\prime}$ of Table 4 we have $(\alpha, \beta)=(r-d \bmod r, d \bmod r)$. In the cases 2 and $2^{\prime}$ we have respectively $(\alpha+\beta, \beta)=(r-d \bmod r, d \bmod r)$ and $(\alpha, \alpha+\beta)=(r-d \bmod r, d \bmod r)$.

Remark 10.2. As in Remark 8.2 for singular Weierstraß curves, for reduced curves we also can interpret the action of small reductions on discrete parameters in terms of the action of Fourier-Mukai transforms. For example, under the condition $\bar{d} \leq r$, the action of the path (13)(12): $A^{+} \rightarrow A^{+}$of the automaton (9.4) and paths (14)(13)(12): $A^{+} \rightarrow A^{+}$of the automatons 10.1) and 10.2) correspond to the action of the Seidel-Thomas twist $\mathrm{T}_{\mathcal{O}}:\left(r, \bar{d}_{1}, \bar{d}_{2}\right) \mapsto\left(r-\bar{d}, \bar{d}_{1}, \bar{d}_{2}\right)$ if $E$ has two components and $\mathrm{T}_{\mathcal{O}}:\left(r, \bar{d}_{1}, \bar{d}_{2}, \bar{d}_{3}\right) \mapsto\left(r-\bar{d}, \bar{d}_{1}, \bar{d}_{2}, \bar{d}_{3}\right)$, for three components. In general a comparison of both actions on rank and multidegree is bulky, and it would not help much for understanding, as far as we have no theorems relating small reductions and Fourier-Mukai transforms on genus one curves. We hope that small reductions $p: \mathfrak{A} \rightarrow \mathfrak{A}^{\prime}$ can be lifted to the level of derived equivalences $p: D^{b}(\mathfrak{A}) \stackrel{\sim}{\longrightarrow} D^{b}\left(\mathfrak{A}^{\prime}\right)$, where $D^{b}(\mathfrak{A})$ is the derived category associated to the box $\mathfrak{A}$, as introduced by Ovsienko in Ovs97. There is a certain analogy with Atiyah's bijections [Ati57] for vector bundles on elliptic curves, which obtained a conceptual explanation in terms of derived categories in LM93, a few decades later after their discovery.

\section{EXAMPLES AND REMARKS}

Example 11.1. Let $E$ be a curve from the list with 2 components, i.e. the Kodaira cycle $\mathrm{I}_{2}$ or the fiber III. Let us describe vector bundles on $E$ of rank $r=9$ and multidegree $\left(d_{1}, d_{2}\right)=(3,2)$ using Algorithm 7.2, The normalization bundle $\widetilde{\mathcal{F}}$ is

$$
\left.\widetilde{\mathcal{F}}\right|_{L_{1}}=\mathcal{O}_{L_{1}}^{6} \oplus\left(\mathcal{O}_{L_{1}}(1)\right)^{3} \text { and }\left.\widetilde{\mathcal{F}}\right|_{L_{2}}=\mathcal{O}_{L_{2}}^{7} \oplus\left(\mathcal{O}_{L_{2}}(1)\right)^{2}
$$

(1) Since $\bar{d}=d=5<9=r$, thus according to Table 2 the input state for the automaton is $A^{+}$and the dimension vector is $\mathbb{s}=\left(s_{1}, s_{2}, s_{3}\right)=(4,2,3)$.

(2) Taking on automaton $(9.3)$ the path $p: A^{+} \stackrel{(12)}{\longrightarrow} B \stackrel{(31)}{\longrightarrow} A^{-} \stackrel{(23)}{\longrightarrow} A^{-} \stackrel{(23)}{\longrightarrow}$ $A^{-} \stackrel{(32)}{\longrightarrow} B \stackrel{(13)}{\longrightarrow} A^{+} \stackrel{(12)}{\longrightarrow} B \stackrel{(31)}{\longrightarrow} A^{-}$we get the reduction of sizes:

$$
\begin{aligned}
(4,2,3) & \stackrel{(12)}{\longmapsto}(2,2,3) \stackrel{(31)}{\longmapsto}(2,2,1) \stackrel{(23)}{\longmapsto}(2,1,1) \stackrel{(23)}{\longmapsto}(2,0,1) \\
& \stackrel{(32)}{\longmapsto}(2,0,1) \stackrel{(13)}{\longmapsto}(1,0,1) \stackrel{(12)}{\longmapsto}(1,0,1) \stackrel{(31)}{\longmapsto}(1,0,0) .
\end{aligned}
$$


Since $s_{2}=0$, reductions $(1,0,1) \stackrel{(12)}{\longmapsto}(1,0,1)$ and $(2,0,1) \stackrel{(32)}{\longmapsto}(2,0,1)$ are degenerated; that is, we formally change states, but the matrices remain the same.

(3) Reversing the path $p$ we construct a canonical form of the matrix $M \in \operatorname{Br}_{\mathfrak{A}}(4,2,3)$.

If $E$ is a Kodaira cycle $\mathrm{I}_{2}$, then

\begin{tabular}{|c|c|c|c|c|c|c|c|c|c|c|c|c|c|c|c|c|}
\hline & & & & & & & & & & & & 1 & & 2 & 3 & \\
\hline & & 1 & 3 & & & & 1 & & 3 & & & 0 & 0 & 0 & 1 & 2 \\
\hline 1 & $(31)(12)$ & 0 & 1 & 1 & $(13)(3$ & & U & & & 1 & 3) & 0 & 1 & 0 & 0 & 1 \\
\hline \begin{tabular}{l|l}
$\lambda$ & 1 \\
\end{tabular} & & 1 & O & $?$ & & & 0 & 0 & 1 & & & 0 & O & 1 & O & \\
\hline & & & & & & & $\lambda$ & 0 & 0 & 3 & & & & & & \\
\hline & & & & & & & & & & & & $\lambda$ & 0 & 0 & 0 & 3 \\
\hline & & & & & & & & & 1 & & 2 & & & 3 & & \\
\hline & & 1 & & 2 & & 3 & & & 0 & 0 & 0 & 0 & 1 & 0 & 0 & 1 \\
\hline & & 0 & 0 & 0 & & 0 & 2 & & 0 & 0 & 0 & 0 & 0 & 1 & 0 & \\
\hline & & 0 & 0 & 0 & 0 & 1 & & & 0 & 0 & 0 & 1 & 0 & 0 & 0 & 2 \\
\hline & 200 & 0 & 1 & 0 & & 0 & 1 & & 0 & 0 & 0 & 0 & 0 & 0 & 1 & \\
\hline & & 0 & 0 & 1 & 0 & 0 & & & 0 & 1 & 0 & 0 & 0 & 0 & 0 & \\
\hline & & $\lambda$ & 0 & 0 & 0 & 0 & 3 & & 0 & 0 & 1 & 0 & 0 & 0 & 0 & 3 \\
\hline & & & & & & & & & $\lambda$ & 0 & 0 & 0 & 0 & 0 & 0 & \\
\hline
\end{tabular}

$\stackrel{(12)}{\longmapsto}$\begin{tabular}{|llll|lll|ll|}
\hline 0 & 0 & 0 & 0 & 1 & 0 & 0 & 0 & 0 \\
0 & 0 & 0 & 0 & 0 & 1 & 0 & 0 & 0 \\
0 & 0 & 0 & 0 & 0 & 0 & 0 & 1 & 0 \\
0 & 0 & 0 & 0 & 0 & 0 & 0 & 0 & 1 \\
\hline 0 & 0 & 0 & 1 & 0 & 0 & 0 & 0 & 0 \\
0 & 0 & 0 & 0 & 0 & 0 & 1 & 0 & 0 \\
\hline 0 & 1 & 0 & 0 & 0 & 0 & 0 & 0 & 0 \\
0 & 0 & 1 & 0 & 0 & 0 & 0 & 0 & 0 \\
$\lambda$ & 0 & 0 & 0 & 0 & 0 & 0 & 0 & 0 \\
\hline
\end{tabular}


Let us construct the canonical form for the tacnode curve. Besides zeros we also use the empty spaces to mark out the blocks $(i j)$, where zeros appear for some general reasons and the corresponding box contains no arrow $j \rightarrow i$. Note that the orders of row and column blocks are chosen in such a way that the matrices have block triangular form (probably with some additional holes).

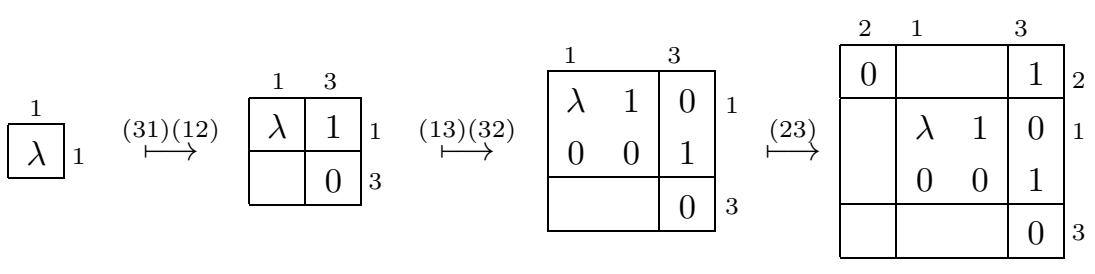

\begin{tabular}{|c|c|c|c|c|c|c|c|c|c|c|}
\hline & & & & & 1 & 2 & & & 3 & \\
\hline & 2 & 1 & 3 & & $\begin{array}{ll}0 & 0\end{array}$ & 0 & 0 & 1 & 0 & 0 \\
\hline & $\begin{array}{ll}0 & 1\end{array}$ & & 0 & 2 & $0 \quad 0$ & 0 & 0 & 0 & 1 & 0 \\
\hline & $\begin{array}{ll}0 & 0\end{array}$ & & 1 & & & 0 & 1 & 0 & 0 & 0 \\
\hline$(23)$ & & $\begin{array}{ll}\lambda & 1\end{array}$ & 0 & $1 \stackrel{(31)}{\longmapsto}$ & & 0 & 0 & 0 & 0 & 1 \\
\hline & & $\begin{array}{ll}0 & 0\end{array}$ & 1 & & & & & $\lambda$ & 1 & 0 \\
\hline & & & 0 & 3 & & & & 0 & 0 & 1 \\
\hline & & & & & & & & 0 & 0 & 0 \\
\hline
\end{tabular}

\begin{tabular}{|llll|lll|ll|}
\hline 0 & 0 & 0 & 0 & 1 & 0 & 0 & 0 & 0 \\
0 & 0 & 0 & 0 & 0 & 1 & 0 & 0 & 0 \\
0 & 0 & 0 & 1 & 0 & 0 & 0 & 1 & 0 \\
0 & 0 & 0 & 0 & 0 & 0 & 1 & 0 & 1 \\
\hline & & & & $\lambda$ & 1 & 0 & & \\
& & & 0 & 0 & 1 & & \\
& & & & 0 & 0 & 0 & & \\
\hline & & & & & & 0 & 0 \\
& & & & & & 0 & 0 \\
\hline
\end{tabular}

Remark 11.2. For a Kodaira fiber II, III and IV the parameter $\lambda$ of the canonical form of $M(\lambda)$ can be moved to any place on the diagonal, as well as it can be distributed as $\frac{\lambda}{r}$ to all the diagonal entries. This way the canonical form resembles 
the Jordan normal form. For instance in the last example we get:

\begin{tabular}{|llll|lll|ll|}
\hline$\frac{\lambda}{9}$ & 0 & 0 & 0 & 1 & 0 & 0 & 0 & 0 \\
0 & $\frac{\lambda}{9}$ & 0 & 0 & 0 & 1 & 0 & 0 & 0 \\
0 & 0 & $\frac{\lambda}{9}$ & 1 & 0 & 0 & 0 & 1 & 0 \\
0 & 0 & 0 & $\frac{\lambda}{9}$ & 0 & 0 & 1 & 0 & 1 \\
\hline & & & & $\frac{\lambda}{9}$ & 1 & 0 & & \\
& & & & 0 & $\frac{\lambda}{9}$ & 1 & & \\
& & & & 0 & 0 & $\frac{\lambda}{9}$ & & \\
\hline & & & & & & & $\frac{\lambda}{9}$ & 0 \\
& & & & & & 0 & $\frac{\lambda}{9}$ \\
\hline
\end{tabular}

12. Properties of Simple VeCtor Bundles

12.1. Tensor products. Let $\mathcal{E}(\lambda) \in \mathrm{VB}_{E}^{s}(r, \mathbb{d})$ and $\mathcal{L}(\lambda) \in \mathrm{Pic}_{E}^{(0, \ldots, 0)}$ be respectively a simple vector bundle and the line bundle with the matrix $M=M(\lambda) \in \mathrm{Br}_{\mathfrak{A}}$ and the parameter $\lambda \in \Lambda$, where $\Lambda:=\mathbb{k}^{*}$ if $E$ is a Kodaira cycle and $\mathbb{k}$ if $E$ is a Kodaira fiber $\left(\Lambda \cong \operatorname{Pic}^{(0, \ldots, 0)}(E)\right)$.

Proposition 12.1. For $\lambda_{1}, \lambda_{2} \in \Lambda$ we have

$$
\mathcal{E}\left(\lambda_{1}\right) \otimes \mathcal{L}\left(\lambda_{2}\right)= \begin{cases}\mathcal{E}\left(\lambda_{1} \cdot \lambda_{2}^{r}\right) & \text { if } E \text { is a Kodaira cycle } I_{1}, I_{2} \text { or } I_{3} \\ \mathcal{E}\left(\lambda_{1}+r \cdot \lambda_{2}\right) & \text { if } E \text { is a Kodaira fiber II, III or IV }\end{cases}
$$

Proof. Let $\left(\widetilde{\mathcal{F}}, V, \tilde{\mu}^{\prime}\left(\lambda_{1}\right)\right)$ and $\left(\widetilde{\mathcal{O}}, \mathcal{O}_{S}, \tilde{\mu}^{\prime \prime}\left(\lambda_{2}\right)\right)$ be the triples of the vector bundle $\mathcal{E}\left(\lambda_{1}\right)$ and the line bundle $\mathcal{L}\left(\lambda_{2}\right)$. Then the triple of the vector bundle $\mathcal{E}\left(\lambda_{1}\right) \otimes_{\mathcal{O}} \mathcal{L}\left(\lambda_{2}\right)$ is $(\widetilde{\mathcal{F}}, V, \tilde{\mu})$, where $\left.\tilde{\mu}:=\tilde{\mu}^{\prime}\left(\lambda_{1}\right) \otimes \tilde{\mu}^{\prime \prime}\left(\lambda_{2}\right)\right)$.

For the Kodaira fiber I: $\mathcal{O}_{S}=\mathbb{k}$ and $\mathcal{O}_{\widetilde{S}}=\mathbb{k} \oplus \mathbb{k}, \tilde{\mu}^{\prime}\left(\lambda_{1}\right)=\left(\mathbb{I}, M\left(\lambda_{1}\right)\right)$ and $\tilde{\mu}^{\prime \prime}\left(\lambda_{2}\right)=\left(1, \lambda_{2}\right)$.

$$
\begin{aligned}
\left.\tilde{\mu}=\tilde{\mu}^{\prime}\left(\lambda_{1}\right) \underset{\mathcal{O}_{\tilde{S}}}{\otimes} \tilde{\mu}^{\prime \prime}\left(\lambda_{2}\right)\right) & =\left(\mathbb{I}, M\left(\lambda_{1}\right) \cdot\left(1, \lambda_{2}\right)\right. \\
& =\left(\mathbb{I}, \lambda_{2} \cdot M\left(\lambda_{1}\right)\right)=\left(\mathbb{I}, M\left(\lambda_{1} \cdot \lambda_{2}^{r}\right)\right) .
\end{aligned}
$$

To obtain the last equality one should reduce $\lambda_{2} \cdot M\left(\lambda_{1}\right)$ to the canonical form preserving the first I-matrix. We illustrate this in the case $r=2$ :

$$
\begin{aligned}
(\tilde{\mu}(0), \tilde{\mu}(\infty)) & =\left(\begin{array}{|c|c}
\hline 1 & 0 \\
\hline 0 & 1
\end{array}, \lambda_{2} \begin{array}{|c|c|}
\hline 0 & 1 \\
\hline \lambda_{1} & 0
\end{array}\right)=\left(\begin{array}{|c|c|c|}
\hline 1 & 0 \\
\hline 0 & 1
\end{array}, \begin{array}{|c|c|c|}
\hline 0 & \lambda_{2} \\
\lambda_{1} \lambda_{2} & 0 \\
\hline \begin{array}{|l|l|}
\lambda_{2} & 0
\end{array} \\
\hline 0 & 1
\end{array}, \begin{array}{|c|c|}
\hline 1 & 0 \\
\hline \lambda_{1} \lambda_{2} & 0 \\
\hline 0 & 1 \\
\hline
\end{array}, \begin{array}{|c|c|}
\hline \lambda_{1} \lambda_{2}^{2} & 0 \\
\hline
\end{array}\right.
\end{aligned}
$$

For the Kodaira fiber II: $\mathcal{O}_{S}=\mathbb{k}$ and $\mathcal{O}_{\widetilde{S}}=\mathbb{k}[\varepsilon] / \varepsilon^{2}, \tilde{\mu}^{\prime}\left(\lambda_{1}\right)=\mathbb{I}+\varepsilon \cdot M\left(\lambda_{1}\right)$ and $\tilde{\mu}^{\prime \prime}\left(\lambda_{2}\right)=1+\varepsilon \cdot \lambda_{2}$.

$$
\begin{aligned}
\left.\tilde{\mu}=\tilde{\mu}^{\prime}\left(\lambda_{1}\right) \underset{\mathcal{O}_{\tilde{S}}}{\otimes} \tilde{\mu}^{\prime \prime}\left(\lambda_{2}\right)\right) & =\left(\mathbb{I}+\varepsilon \cdot M\left(\lambda_{1}\right)\right) \cdot\left(1+\varepsilon \cdot \lambda_{2}\right) \\
& =\mathbb{I}+\varepsilon \cdot\left(M\left(\lambda_{1}\right)+\lambda_{2} \cdot \mathbb{I}\right)=\mathbb{I}+\varepsilon \cdot M\left(\lambda_{1}+\lambda_{2}\right) .
\end{aligned}
$$


The last equality follows immediately if we rewrite $M(\lambda)$ in the "diagonal" form (11.1). For example, if $r=2$ and $d=1$ we have

$$
\tilde{\mu}=\tilde{\mu}(0)+\varepsilon \tilde{\mu}_{\varepsilon}(0)=\begin{array}{|c|c|}
\hline 1 & 0 \\
\hline 0 & 1 \\
\hline
\end{array}+\varepsilon \cdot\left(\begin{array}{c|c|c|c|}
\hline \frac{\lambda_{1}}{2} & 1 \\
\hline 0 & \frac{\lambda_{1}}{2} \\
\hline
\end{array}+\begin{array}{|c|c|c|}
\hline \lambda_{2} & 0 \\
\hline 0 & \lambda_{2} \\
\hline 1 & 0 \\
\hline 0 & 1 \\
\hline
\end{array}+\varepsilon \begin{array}{c|c|c|}
\hline \frac{\lambda_{1}+2 \lambda_{2}}{2} & 1 \\
\hline 0 & \frac{\lambda_{1}+2 \lambda_{2}}{2} \\
\hline
\end{array} .\right.
$$

For Kodaira cycles $\mathrm{I}_{2}, \mathrm{I}_{3}$ and fibers III and IV the calculations should be carried out on each component. On the first component the picture is similar to the cases of I and II. On the other components we have $\tilde{\mu}_{k}=\tilde{\mu}_{k}^{\prime}$.

Example 12.2. If $r=3$ and $\mathbb{d}=(1,1)$ for Kodaira cycle $\mathrm{I}_{2}$ we have

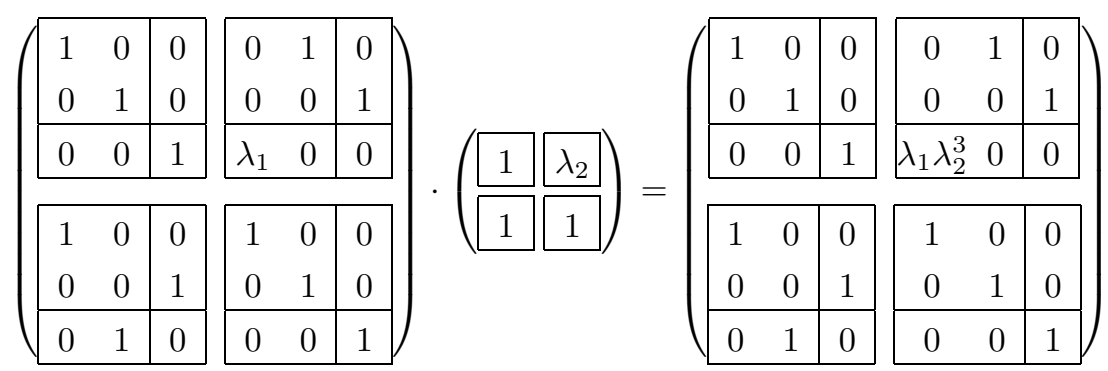

and for Kodaira fiber III taking $\lambda:=\lambda_{1}+3 \lambda_{2}$ we have

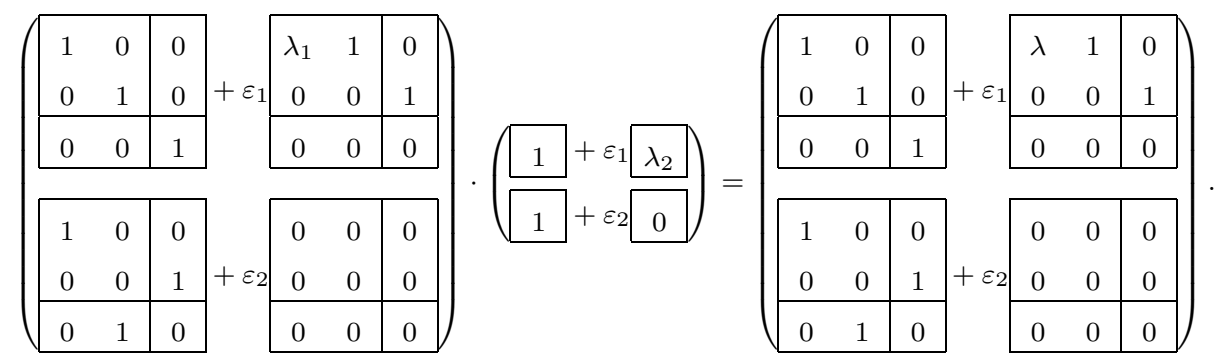

\subsection{Morphisms.}

Proposition 12.3. Let $E$ be one of the curves from Table 1 and $\mathcal{E}\left(\lambda_{1}\right), \mathcal{E}\left(\lambda_{2}\right) \in$ $\operatorname{VB}_{E}^{s}(r, \mathbb{d})$ with $\lambda_{1} \neq \lambda_{2}$. Then $\operatorname{Hom}_{E}\left(\mathcal{E}\left(\lambda_{1}\right), \mathcal{E}\left(\lambda_{2}\right)\right)=0$.

Proof. From the equivalence $\mathrm{VB}_{E}^{s}(r, \mathbb{d}) \stackrel{\sim}{\rightarrow} \mathrm{Br}_{\mathfrak{A}}(\mathrm{s})$ we have:

$$
\operatorname{Hom}_{E}\left(\mathcal{E}\left(\lambda_{1}\right), \mathcal{E}\left(\lambda_{2}\right)\right)=\operatorname{Hom}_{\mathfrak{A}}\left(M\left(\lambda_{1}\right), M\left(\lambda_{2}\right)\right) .
$$

Let $\left(S, S^{\prime}\right) \in \operatorname{Hom}_{\mathfrak{A}}\left(M\left(\lambda_{1}\right), M\left(\lambda_{2}\right)\right)$. If $r=1$ and $\left(S, S^{\prime}\right) \neq 0$, then $S^{\prime}=S \in \mathbb{k}^{*}$ and since $M\left(\lambda_{1}\right)=\lambda_{1}$, and $M\left(\lambda_{2}\right)=\lambda_{2}$, we get a contradiction: $S \lambda_{1} S^{-1}=\lambda_{2}$. Recall that a path $p$ on a small reduction automaton gives an equivalence of the categories $\mathrm{Br}_{\mathfrak{A}}(\mathbb{s}) \stackrel{p}{\longrightarrow} \operatorname{Br}_{\mathfrak{A}^{\prime}}\left(\mathbb{s}^{\prime}\right)$, where $\mathbb{s}^{\prime} \leq \mathbb{s}$. Thus the statement follows by induction on the dimension vector $s$ along the path $p$.

Remark 12.4. By the same approach one can also describe torsion-free sheaves which are not vector bundles. We are going to consider this situation in further works. One can consult [Bod07], Sections 3.3, 4.5 and 7.7, about torsion-free sheaves on cuspidal and tacnode curves. 


\section{REFERENCES}

[AK79] Altman, A., Kleiman, S.: Compactifying of the Picard scheme II. Amer. J. Math. 101, 10-41 (1979). MR 527824 (81f:14025b)

[Ati57] Atiyah, M.: Vector bundles over an elliptic curve. Proc. London Math. Soc., 7, 414452 (1957). MR0131423 (24:A1274)

[BBHM02] Bartocci, C., Bruzzo, U., Hernández Ruipérez, D., Muñoz Porras, J.: Relatively stable bundles over elliptic fibrations. Math. Nachr. 238, 23-36 (2002). MR1900809 (2003m:14019)

[BPV84] Barth, W., Peters, C., Van de Ven, A., Compact complex surfaces, Ergebnisse der Mathematik und ihrer Grenzgebiete (3), Springer-Verlag, Berlin, 1984. MR749574 (86c:32026)

[BBDG] Bodnarchuk, L., Burban, I., Drozd, Yu., Greuel, G.-M.: Vector bundles and torsionfree sheaves on degenerations of elliptic curves, Global Aspects of Complex Geometry 83128, (2006), arxiv: math.AG/16796. MR.2264108(2008a:14048)

[BD03] Bodnarchuk, L., Drozd, Yu. A.: Stable vector bundles over cuspidal cubics, Central European Journal of Mathematics 4, 650-660, (2003). MR2040656 (2005d:14047)

[BD09] Bodnarchuk, L., Drozd, Yu. A.: One class of wild but brick-tame matrix problems preprint MPIM2009-18, arXiv: math.RT /0903.4374v2.

[BB91] Butler, M.C.R., Burt, W.L.: Almost split sequences for bocses. Canad. Math. Soc. Conf. Proc. 11, 89-121 (1991). MR:1143847 (93d:16017)

[BD04] Burban, I., Drozd, Yu.: Coherent sheaves on rational curves with simple double points and transversal intersections. Duke Math. J. 121, no. 2, 189-229 (2004). MR2034641 (2004m:14024)

[BD09] Burban, I., Drozd, Yu.: Tilting on non-commutative rational projective curves, arXiv:0905.1231 (to appear in Mat. Annalen).

[BDG01] Burban, I., Drozd, Yu., Greuel, G.-M.: Vector bundles on singular projective curves. Ciliberto (ed.) et al., Applications of algebraic geometry to coding theory, physics and computation (Eilat, Israel, 2001), Dordrecht: Kluwer Academic Publishers, NATO Sci. Ser. II, Math. Phys. Chem. 36, 1-15 (2001). MR1866891 (2002k:14054)

[BK05] Burban, I., Kreußler, B.: Fourier-Mukai transforms and semi-stable sheaves on nodal Weierstraß cubics. J. Reine Angew. Math., 584, 45-82 (2005). MR2155085 (2006d:14016)

[BK06] Burban, I., Kreußler, B.: Derived categories of irreducible projective curves of arithmetic genus one. Compositio Mathematica, 142, 1231-1262 (2006). MR2264663 (2007h:18016)

[BK4] Burban, I., Kreußler, B.: Vector bundles on cubic curves and Yang-Baxter equations. arxiv: math.AG/0708.1685v2.

[Bho92] Bhosle, U.N.: Generalised parabolic bundles and applications to torsionfree sheaves on nodal curves, Ark. Mat 30, 187-215 (1992). MR.1289750 (95g:14022)

[Bho96] Bhosle, U.N.: Generalized parabolic bundles and applications II. Proc. Indian Acad. Sci. (Math. Sci.) 106, N4 403-420 (1996). MR1425615 (2000i:14048)

[Bho99] Bhosle, U.N.: Moduli of vector bundles on curves with many components. Proceedings of the London Mathematical Society, Cambridge University Press 79, 81-106 (1999). MR:1687547 (2000c:14043)

[Bod07] Bodnarchuk, L.: Simple vector bundles on degenerations of elliptic curves of type II, III and IV. Ph.D. thesis, Kaiserslautern (2007) http://kluedo.ub.unikl.de/volltexte/2008/2281/.

[Bon92] Bondarenko, V. M.: Representations of bundles of semi-chains and their applications. St. Petersburg Math. J., 3, 973-996 (1992). MR.1186235 (94h:16020)

[Bur03] Burban, I.: Stable vector bundles on a rational curve with one simple node. Ukrainian Mathematical Journal 5, (2003).

[CB88] Crawley-Boevey, W.: On tame algebras and bocses. Proc. London Math. Soc. 56, 451-483 (1988). MR.931510 (89c:16028)

[CB89] Crawley-Boevey, W.: Functorial filtrations II: clans and the Gelfand problem. J. London Math. Soc., 40, 9-30 (1989). MR1028911(91i:16025) 
[CB90] Crawley-Boevey, W.: Matrix problems and Drozd's theorem. Topics in Algebra, Balcerzyk, S., et al., eds., Banach Center publications, vol. 26 part 1 (PWN-Polish Scientific Publishers, Warsaw), 199-222 (1990). MR.1171233 (93g:16021)

[DG01] Drozd, Yu., Greuel, G.-M.: Tame and Wild Projective Curves and Classification of Vector Bundles. Journal of Algebra 246, 1-54 (2001). MR1872612 (2002j:14039)

[Dro79] Drozd, Yu.: Tame and wild matrix problems. Representations and Quadratic Forms. Institute of Mathematiks, Kiev, 39-74 (1979) (English translation: Amer. Math. Soc. Transl. 128, 31-55 (1986)). MR600111 (82m:16028)

[Dro92] Drozd, Yu.: Matrix problems, small reduction and representations of a class of mixed Lie groups. Representations of Algebras and Related Topics. Cambridge Univ. Press, 225-249 (1992). MR1211482 (94d:22016)

[Dro01] Drozd, Yu.: Reduction Algorithm and representations of boxes and algebras. C.R. Math. Pep. Acad. Sci. Canada, 23, 97-125 (2001). MR.1869054 (2002j:16012)

[Dro05] Drozd, Yu.: Semi-continuity for derived categories. Algebras and Representation Theory, 8 239-248 (2005). MR2162284 (2006f:16022)

[FMW99] Friedman, R., Morgan, J., Witten, E.: Vector bundles over elliptic fibrations. J. Algebr. Geom. 8, 279-401 (1999). MR1675162 (2000b:14053)

[HLSP] Hernández Ruipérez, D., López Martin, A.C., Sánchez Gómez, D.S., Prieto, C.T.: Moduli spaces of semistable sheaves on singular genus one curves. Int. Math. Res. Notices 11, 4428-4462 (2009), DOI 10.1093/imrn/rnp094, arXiv:math.AG/0806.2034.

[GP68] Gelfand, I., Ponomarev, V.: Indecomposable representations of the Lorentz group. Uspehi Mat. Nauk 23, no. 2 140, 3-60 (1968). MR0229751(37:5325)

[KL86] Klinger, L., Levy, L.S.: Sweeping-similarity of matrices. Linear algebra and its applications 75, 67-104 (1986). MR825400 (87k:15015)

[KR75] Kleiner, M.M., Roiter, A.V.: Representations of differential graded categories. Representations of Algebras. Proc.Conf. Ottawa (1974) (eds.) V. Dlab and P.Gabriel. Lecture Notes in Math. 488, Springer, Berlin, 316-339 (1975). MR 0435145 (55:8106)

[Kri77] Krichever, I. M.: Integration of nonlinear equations by the methods of algebraic geometry. (Russian) Funkcional. Anal. i Prilozhen. 11, no. 1, 15-31 (1977). MR0494262 $(58: 13168)$

[LM93] Lenzing, H. and Meltzer, H.: Sheaves on a weighted projective line of genus one, and representations of a tubular algebra. Can. Math. Soc. Conf. Proc. 14, 313- 337 (1993).

[Lo05] López-Martin, A.-C.: Simpson Jacobians of reducible curves, J. reine angew. Math. 582, 1-39 (2005). MR2139709 (2006d:14032)

[Lo06] López-Martin, A.-C.: Relative Jacobians of elliptic fibrations with reducible fibers. Journal of Geometry and Physics 56, 375-385 (2006). MR2171891 (2006g:14020)

[Ma78] Manin, Yu. I.: Matrix solitons and vector bundles over curves with singularities. (Russian) Funk. Anal. i Prilozhen. 12, no. 4, 53-63 (1978). MR515629 (80g:35114)

[Mu94] Mulase, M.: Algebraic theory of the KP equations. Perspectives in Mathematical Physics, Editors R. Penner and S.-T. Yau, International Press Company, 157-223 (1994). MR1314667 (96c:58088)

[NR69] Nazarova, L.A., Roiter, A.V.: Finitely generated modules over a dyad of two local Dedekind rings, and finite groups with an Abelian normal divisor of index $p$. Math. USSR Izv., 3, 65-86 (1969); translation from Izv. Akad. Nauk SSSR, Ser. Mat., 33, 65-89 (1969).

[New78] Newstead, P.: Introduction to Moduli Problems and Orbit Spaces. T.I.F.R. Lecture Notes 51 (1978). MR546290(81k:14002)

[Ovs97] Ovsienko, S.: Bimodule and matrix problems. Euroconference Essen Computational Methods for Representations of Groups and Algebras, April 1 - 5, (1997).

[Pol02] Polishchuk, A.: Classical Yang-Baxter equation and the $A_{\infty}$-constraint. Adv. Math. 168, no. 1, 56-95 (2002). MR.1907318(2003d:16051)

[Pol07] Polishchuk, A.: Massey products on cycles of projective lines and trigonometric solutions of the Yang-Baxter equations. arXiv:math/0612761v3.

[Ro79] Roiter A. V.: Matrix problems and representations of BOCSs. Representation Theory I, Proc. Conf. Ottawa 1979, Dlab V. and Gabriel P. (eds.), Lecture Notes in Math. 831, 288-324, Springer, Berlin (1980). MR607144 (83e:16034) 
[Ro86] Roiter A. V.: Matrix problems and representations of BOCSs. Representations and Quadratic Forms. Institute of Mathematiks, Kiev, 39-74 (1979) (English translation: Amer. Math. Soc. Transl. 128, 31-55 (1986)). MR600110(83b:16027)

[ST01] Seidel, P., Thomas, R.P.: Braid group actions on derived categories of coherent sheaves. Duke Math. J., 108, no. 1, 37-108 (2001). MR1831820(2002e:14030)

[Ses82] Seshadri, C.S.: Fibrés vectoriels sur les courbes algébriques. Astérisque, 96, Société Mathématique de France, Paris (1982). MR699278 (85b:14023)

Max-Planck-Institut für Mathematik, Bonn, Germany

E-mail address: lesyabod@mpim-bonn.mpg.de

Institute of Mathematics, National Academy of Sciences of Ukraine, Kiev, Ukraine E-mail address: drozd@imath.kiev.ua

Fachbereich Mathematik, University of Kaiserslautern, Kaiserslautern, Germany

E-mail address: greuel@mathematik.uni-kl.de 